\title{
Formal Total Synthesis of Optically Active Ingenol via Ring-Closing Olefin Metathesis
}

Kazushi Watanabe, Yuto Suzuki, Kenta Aoki, Akira Sakakura, Kiyotake Suenaga, and Hideo

\author{
Kigoshi*
}

Department of Chemistry, University of Tsukuba, Tennoudai, Tsukuba, Ibaraki 305-8571,

Japan

\section{Supporting Information}

Table of contents

General procedures

Experimental procedures for compounds 3a, 11, 12, 14, 16, 17, 18a, 18b, 19a, 19b, 4, 20,
S1

S2

5, 21, 22a, and 22b

S2-S8

${ }^{1} \mathrm{H}$ and $/$ or ${ }^{13} \mathrm{C}$ NMR charts for 16, 17, 18a, 18b, 19a, 19b, 4, 20, 5, 21, 22a, 22b, 23, 24, 25, 26, 28, 29, 30, 7, 8, and 9

S9-S39 
General Procedures. Melting points are uncorrected. NMR chemical shifts were referenced to solvent peaks: $\delta_{\mathrm{H}} 7.26$ (residual $\mathrm{CHCl}_{3}$ ) for $\mathrm{CDCl}_{3}, \delta_{\mathrm{C}} 77.1$ for $\mathrm{CDCl}_{3}$, and $\delta_{\mathrm{C}} 128.0$ for benzene- $d_{6}$. Mass spectra were determined on a FAB mode ( $m$-nitrobenzyl alcohol as a matrix). High resolution mass spectra (HRMS) were recorded on a ESI mode, a FAB mode ( $m$-nitrobenzyl alcohol as a matrix), or the EI mode. Unless otherwise stated, materials were obtained from commercial suppliers and used without further purification. All organic solvents and reagents were purified by standard procedures. All moisture-sensitive reactions were performed under an atmosphere of nitrogen.

Bicyclic ketone 3a [40863-62-5]. A solution of cis-diallylcycloheptanone (50.3 mg, 0.27 $\mathrm{mmol})$ and the first generation Grubbs catalyst, $\mathrm{RuCl}_{2}(=\mathrm{CHPh})\left(\mathrm{PCy}_{3}\right)_{2}(18 \mathrm{mg}, 0.025 \mathrm{mmol})$ in benzene was refluxed for $18 \mathrm{~h}$. The mixture was concentrated to give a oil, which was chromatographed on silica gel (5 g, hexane-ether 30:1 to 5:1) to give bicyclic ketone 3a (19.9 $\mathrm{mg}, 45 \%)$ as a colorless oil. IR $\left(\mathrm{CHCl}_{3}\right)$ 3080, 3000, 2930, 2845, 1695, 1640, 1455, 1360, 910 $\mathrm{cm}^{-1}$; ${ }^{1} \mathrm{H} \mathrm{NMR}\left(270 \mathrm{MHz}, \mathrm{CDCl}_{3}\right) \delta 5.55(\mathrm{~m}, 2 \mathrm{H}), 2.77$ (tt, $\left.J=6.9,7.3 \mathrm{~Hz}, 2 \mathrm{H}\right), 2.4$ (ddd, $J$ $=15.5,6.9,1.3 \mathrm{~Hz}, 2 \mathrm{H}), 2.30-2.10(\mathrm{~m}, 2 \mathrm{H}), 1.98-1.74(\mathrm{~m}, 6 \mathrm{H}), 1.55-1.42(\mathrm{~m}, 2 \mathrm{H}) ;{ }^{13} \mathrm{C}$ NMR $\left(67.8 \mathrm{MHz}, \mathrm{CDCl}_{3}\right) \delta 217.0,127.8,53.7,30.3,28.9,25.7$; MS (GCEI) $\mathrm{m} / \mathrm{z} .164\left(\mathrm{M}^{+}\right.$, $100)$.

Conjugated ester 11 [119729-72-5]. To a cooled $\left(-78^{\circ} \mathrm{C}\right)$ solution of $\gamma$-butyrolactone $(2.24 \mathrm{~g}$, $26.0 \mathrm{mmol})$ in toluene $(50 \mathrm{~mL})$ was added a $1.0 \mathrm{M}$ solution of DIBAL in toluene $(39 \mathrm{~mL}, 39$ mmol), and the mixture was stirred at same temperature for $5 \mathrm{~h}$. After the reaction was quenched by addition of methanol $(3.3 \mathrm{~mL})$, the mixture was warmed to $0{ }^{\circ} \mathrm{C}$ and diluted with water $(8.8 \mathrm{~mL})$. After the mixture was stirred with $\mathrm{MgSO}_{4}(8.8 \mathrm{~g})$ and Celite $(8.8 \mathrm{~g})$ for 20 min, the mixture was filtered through a pad of Celite, and the filtered residue was washed with ether. The filtrate and washings were combined and concentrated to give a crude hemiacetal $(2.2 \mathrm{~g})$ as a colorless oil. This material was used for the next reaction without further purification. IR $\left(\mathrm{CHCl}_{3}\right)$ 3600, 3410 (br), 3010, 2980, 2960, 2880, 1770, 1720, 1460, 1440, 1400, 1365, $1340 \mathrm{~cm}^{-1}$; ${ }^{1} \mathrm{H}$ NMR (270 MHz, $\left.\mathrm{CDCl}_{3}\right) \delta 5.54(\mathrm{~m}, 1 \mathrm{H}), 4.03(\mathrm{~m}, 1 \mathrm{H})$, 3.86 (m, 2 H), 1.93-1.85 (m, $4 \mathrm{H})$.

A solution of hemiacetal (2.2 g) and methyl triphenylphosphoranylideneacetate $(12.4 \mathrm{~g}, 37.1$ mmol) in benzene $(50 \mathrm{~mL})$ was stirred at $60{ }^{\circ} \mathrm{C}$ for $4 \mathrm{~h}$ and cooled to room temperature. The mixture was diluted with hexane-ether $(1: 1,170 \mathrm{~mL})$ and filtered through a pad of Celite. The filtered residue was washed with hexane-ether (1:1), and the filtrate and washings were 
combined and concentrated. The residue was chromatographed on silica gel (100 g, benzeneEtOAc 30:1) to give conjugated ester $11(2.21 \mathrm{~g}, 59 \%)$ as a colorless oil. ${ }^{1} \mathrm{H}$ NMR $(270 \mathrm{MHz}$, $\left.\mathrm{CDCl}_{3}\right) \delta 6.98(\mathrm{dt}, J=15.7,7.0 \mathrm{~Hz}, 1 \mathrm{H}), 5.86(\mathrm{dt}, J=15.7,1.6 \mathrm{~Hz}, 1 \mathrm{H}), 3.73(\mathrm{~s}, 3 \mathrm{H}), 3.68(\mathrm{t}$, $J=6.1 \mathrm{~Hz}, 2 \mathrm{H}), 2.31(\mathrm{tdd}, J=7.0,7.0,1.6 \mathrm{~Hz}, 2 \mathrm{H}), 1.73(\mathrm{tt}, J=7.0,6.1 \mathrm{~Hz}, 2 \mathrm{H}), 1.56$ (br s, $1 \mathrm{H})$.

Iodide 12 [77823-74-6]. To a stirred solution of conjugated ester 11 (1.19 g, 8.3 mmol), triphenyphosphine (3.46 g, $13.2 \mathrm{mmol})$, and imidazole $(1.70 \mathrm{~g}, 25.0 \mathrm{mmol})$ was added iodine $(3.80 \mathrm{~g}, 14.9 \mathrm{mmol})$, and the mixture was vigorously stirred under dark for $1.5 \mathrm{~h}$. The mixture was diluted with saturated aqueous $\mathrm{NaHCO}_{3}(35 \mathrm{~mL})$ and saturated aqueous $\mathrm{Na}_{2} \mathrm{~S}_{2} \mathrm{O}_{3}(14 \mathrm{~mL})$ and extracted with EtOAc $(3 \times 60 \mathrm{~mL})$. The combined extracts were washed with brine, dried, and concentrated. The residue was chromatographed on silica gel (100 g, hexane-EtOAc 8:1 to 5:1) to give iodide $12(1.71 \mathrm{~g}, 81 \%)$ as a colorless oil. IR $\left(\mathrm{CHCl}_{3}\right)$ 3000, 2985, 2930, 2880, 1710, 1660, 1490, 1440, 1380, 1370, $1280 \mathrm{~cm}^{-1}$; ${ }^{1} \mathrm{H}$ NMR $\left(270 \mathrm{MHz}, \mathrm{CDCl}_{3}\right) \delta 6.90(\mathrm{dt}, J=$ 15.7, 7.0 Hz, $1 \mathrm{H}), 5.89$ (dt, $J=15.7,1.6 \mathrm{~Hz}, 1 \mathrm{H}), 3.73(\mathrm{~s}, 3 \mathrm{H}), 3.19$ (t, $J=6.9 \mathrm{~Hz}, 2 \mathrm{H})$, $2.34(\mathrm{tdd}, J=7.0,7.0,1.6 \mathrm{~Hz}, 2 \mathrm{H}), 1.98(\mathrm{tt}, J=7.0,6.9 \mathrm{~Hz}, 2 \mathrm{H}) ;{ }^{13} \mathrm{C} \mathrm{NMR}(67.8 \mathrm{MHz}$, $\left.\mathrm{CDCl}_{3}\right) \delta 166.8,146.9,122.2,51.5,32.7,31.4,5.4$.

THP ether 14 [83632-97-7]. To a cooled $\left(-78^{\circ} \mathrm{C}\right)$ solution of iodide $12(1.71 \mathrm{~g}, 6.74 \mathrm{mmol})$ in toluene $(40 \mathrm{~mL})$ was added a $0.95 \mathrm{M}$ solution of DIBAL in hexane $(15.6 \mathrm{~mL}, 14.8 \mathrm{mmol})$, and the mixture was stirred at same temperature for $1 \mathrm{~h}$. After the reaction was quenched by addition of methanol $(1 \mathrm{~mL})$, the mixture was warmed to $0{ }^{\circ} \mathrm{C}$ and diluted with water $(2 \mathrm{~mL})$. After the mixture was stirred with $\mathrm{MgSO}_{4}(3 \mathrm{~g})$ and Celite $(3 \mathrm{~g})$ for $20 \mathrm{~min}$, the mixture was filtered through a pad of Celite, and the filtered residue was washed with ether. The filtrate and washings were combined and concentrated to give a crude alcohol $13(1.54 \mathrm{~g})$ as a colorless oil. This material was used for the next reaction without further purification. IR $\left(\mathrm{CHCl}_{3}\right)$ 3610, 3450 (br) , 3005, 2930, 2850, 1670, 1430, 1380, $1260 \mathrm{~cm}^{-1} ;{ }^{1} \mathrm{H}$ NMR (270 $\left.\mathrm{MHz}, \mathrm{CDCl}_{3}\right) \delta 5.70(\mathrm{dt}, J=15.4,4.6 \mathrm{~Hz}, 1 \mathrm{H}), 5.67(\mathrm{dt}, J=15.4,5.0 \mathrm{~Hz}, 1 \mathrm{H}), 4.10(\mathrm{~d}, J=$ $4.6 \mathrm{~Hz}, 2 \mathrm{H}$ ), 3.19 (t, $J=6.9 \mathrm{~Hz}, 2 \mathrm{H}$ ), 2.18 (m, $2 \mathrm{H}$ ), 1.91 (quint, $J=6.9 \mathrm{~Hz}, 2 \mathrm{H}$ ), 1.43 (s, 1 $\mathrm{H}) ;{ }^{13} \mathrm{C}$ NMR $\left(67.8 \mathrm{MHz}, \mathrm{CDCl}_{3}\right) \delta 130.5,63.5,32.7,32.6,6.2$.

A solution of the crude alcohol 13 (1.54 g), 3,4-dihydropyran (1.2 $\mathrm{mL}, 13.3 \mathrm{mmol})$, and $p$ toluenesulfonic acid $(4.4 \mathrm{mg}, 0.023 \mathrm{mmol})$ in $\mathrm{CH}_{2} \mathrm{Cl}_{2}(20 \mathrm{~mL})$ was stirred at room temperature for $40 \mathrm{~min}$. The mixture was diluted with saturated aqueous $\mathrm{NaHCO}_{3}(30 \mathrm{~mL})$ and extracted with ether $(3 \times 20 \mathrm{~mL})$. The combined extracts were washed with brine, dried, 
and concentrated. The residue was chromatographed on silica gel. (50 g, hexane-EtOAc 20:1 to 10:1) to give THP ether $14(1.96 \mathrm{~g}, 94 \%)$ as a colorless oil. IR $\left(\mathrm{CHCl}_{3}\right)$ 3005, 2960, 2870, 2850, 1455, 1440, 1380, 1350, 1320, $1260 \mathrm{~cm}^{-1} ;{ }^{1} \mathrm{H}$ NMR (270 MHz, $\left.\mathrm{CDCl}_{3}\right) \delta 5.66(\mathrm{~m}, 2 \mathrm{H})$, $4.63(\mathrm{t}, J=3.4 \mathrm{~Hz}, 1 \mathrm{H}), 4.19(\mathrm{~m}, 1 \mathrm{H}), 3.96-3.88(\mathrm{~m}, 2 \mathrm{H}), 3.51(\mathrm{~m}, 1 \mathrm{H}), 3.19$ (t, $J=6.9 \mathrm{~Hz}$, $2 \mathrm{H}), 2.17(\mathrm{~m}, 2 \mathrm{H}), 1.92(\mathrm{tt}, J=7.2,6.9 \mathrm{~Hz}, 2 \mathrm{H}), 1.85-1.53(\mathrm{~m}, 6 \mathrm{H}) ;{ }^{13} \mathrm{C} \mathrm{NMR}(67.8 \mathrm{MHz}$, $\left.\mathrm{CDCl}_{3}\right) \delta 131.7,127.8,97.9,67.6,62.3,32.9,32.6,30.7,25.5,19.5,6.3 ; \mathrm{MS}(\mathrm{FAB}) \mathrm{m} / \mathrm{z} 333$ $(\mathrm{M}+\mathrm{Na})^{+}$.

Alkylated cycloheptanone 16. To a solution of cycloheptanone $N, N$-dimethylhydrazone $(0.84 \mathrm{~mL}, 860 \mathrm{mg}, 5.57 \mathrm{mmol})$ in THF $(5 \mathrm{~mL})$ was added a $1.6 \mathrm{M}$ solution of $n$-BuLi in hexane $(3.91 \mathrm{~mL}, 6.15 \mathrm{mmol})$ at $-5^{\circ} \mathrm{C}$. After being stirred for $1 \mathrm{~h}$, a solution of THP ether 14 $(1.65 \mathrm{~g}, 5.30 \mathrm{mmol})$ in THF $(1.5+0.5 \mathrm{~mL})$ was added. After being stirred at room temperature for $1 \mathrm{~h}$, the mixture was diluted with water and extracted with EtOAc $(3 \times 15$ $\mathrm{mL}$ ). The combined extracts were washed with saturated aqueous $\mathrm{Na}_{2} \mathrm{~S}_{2} \mathrm{O}_{3}$ and brine, successively; dried; and concentrated. The residue was diluted with $\mathrm{CH}_{2} \mathrm{Cl}_{2}$ (60 mL) and stirred with silica gel (16 g) for $19 \mathrm{~h}$. The mixture was filtered through a cotton plug, and the silica gel was eluted with ether. The filtrate and washings were combined and concentrated to give a oil, which was chromatographed on silica gel (40 g, hexane-ether 2:1) to give the desired alkylated cycloheptanone $16(1.16 \mathrm{~g}, 74 \%)$ and its $N, N$-dimethylhydrazone 15 (405 $\mathrm{mg}, 24 \%)$ as a colorless oil, respectively.

Hydrolysis of the hydrazone 15 (405 mg) with silica gel (4 g) in $\mathrm{CH}_{2} \mathrm{Cl}_{2}(16 \mathrm{~mL})$ afforded the desired ketone 16 (283 mg, 18\% from cycloheptanone $N, N$-dimethylhydrazone). IR ( $\left.\mathrm{CHCl}_{3}\right)$ 3005, 2940, 2850, 1695, 1455, 1380, 1350, 1320, $1260 \mathrm{~cm}^{-1} ;{ }^{1} \mathrm{H}$ NMR $\left(270 \mathrm{MHz}, \mathrm{CDCl}_{3}\right) \delta$ 5.74-5.51 (m, $2 \mathrm{H}), 4.63$ (br t, $J=3.5 \mathrm{~Hz}, 1 \mathrm{H}), 4.18$ (ddd, $J=12.0,5.6,1.0 \mathrm{~Hz}, 1 \mathrm{H}), 3.91$ (br dd, $J=12.0,7.3 \mathrm{~Hz}, 1 \mathrm{H}), 3.86(\mathrm{~m}, 1 \mathrm{H}), 3.49$ (m, $1 \mathrm{H}), 2.55-2.38$ (m, $3 \mathrm{H}), 2.03$ (br q, $J$ $=6.6 \mathrm{~Hz}, 2 \mathrm{H}), 1.84-1.33(\mathrm{~m}, 18 \mathrm{H})$; HRMS (FAB) $m / z$ calcd. for $\mathrm{C}_{18} \mathrm{H}_{30} \mathrm{NaO}_{3}(\mathrm{M}+\mathrm{Na})^{+}$ 317.2093, found $317.2089(\Delta-0.4 \mathrm{mmu})$.

Allylic chloride 17. A solution of the alkylated cycloheptanone 16 (610 mg, $2.07 \mathrm{mmol})$, pyridinium $p$-toluenesulfonate $(42 \mathrm{mg}, 0.17 \mathrm{mmol})$ in ethanol $(40 \mathrm{~mL})$ was stirred at $55^{\circ} \mathrm{C}$ for $12 \mathrm{~h}$. The reaction mixture was concentrated, and the residue was chromatographed on silica gel (30 g, hexane-ether 2:1) to give an allylic alcohol (422 $\mathrm{mg}, 97 \%)$ as a colorless oil. IR $\left(\mathrm{CHCl}_{3}\right)$ 3605, 3480, 3020, 2930, 2850, 1710, 1455, 1360, 1225, 1090, $970 \mathrm{~cm}^{-1} ;{ }^{1} \mathrm{H}$ NMR $\left(270 \mathrm{MHz}, \mathrm{CDCl}_{3}\right) \delta 5.64(\mathrm{~m}, 2 \mathrm{H}), 4.07(\mathrm{~m}, 2 \mathrm{H}), 2.50-2.45(\mathrm{~m}, 3 \mathrm{H}), 2.00(\mathrm{~m}, 1 \mathrm{H}), 1.84(\mathrm{~m}$, 
$4 \mathrm{H}), 1.66$ (m, $2 \mathrm{H}), 1.35$ (m, 8 H). MS (EI, intensity) m/z $210\left(\mathrm{M}^{+}, 17\right), 192$ (6), 112 (100).

The solution of the allylic alcohol $(313 \mathrm{mg}, 1.49 \mathrm{mmol})$ and $\mathrm{PPh}_{3}(780 \mathrm{mg}, 2.97 \mathrm{mmol})$ in $\mathrm{CCl}_{4}(10 \mathrm{~mL})$ was refluxed for $5 \mathrm{~h}$. Triphenylphosphine (390 mg, $1.49 \mathrm{mmol}$ ) was added, and the mixture was refluxed for further $4 \mathrm{~h}$. After being cooled to room temperature, the reaction mixture was diluted with hexane $(10 \mathrm{~mL})$ and filtered through a cotton plug. The residue was washed with hexane, and the filtrate and washings were combined and concentrated. The residue was chromatographed on silica gel (10 g, hexane-ether 25:1) to give allylic chloride $17(317 \mathrm{mg}, 93 \%)$ as a colorless oil. IR $\left(\mathrm{CHCl}_{3}\right) 2928,2855,1700,1457,1250,966 \mathrm{~cm}^{-1} ;{ }^{1} \mathrm{H}$ NMR (270 MHz, $\left.\mathrm{CDCl}_{3}\right) \delta 5.74(\mathrm{dt}, \mathrm{J}=14.9,6.9 \mathrm{~Hz}, 1 \mathrm{H}), 5.60(\mathrm{dt}, \mathrm{J}=14.9,6.9 \mathrm{~Hz}, 1 \mathrm{H})$, $4.02(\mathrm{~d}, \mathrm{~J}=6.9 \mathrm{~Hz}, 2 \mathrm{H}), 2.55-2.39(\mathrm{~m}, 3 \mathrm{H}), 2.05(\mathrm{dt}, \mathrm{J}=6.9,6.8 \mathrm{~Hz}, 2 \mathrm{H}), 1.88-1.83$ (m, 4 $\mathrm{H}), 1.75-1.58(\mathrm{~m}, 2 \mathrm{H}), 1.41-1.31(\mathrm{~m}, 6 \mathrm{H})$; HRMS (FAB) $\mathrm{m} / \mathrm{z}$ calcd. for $\mathrm{C}_{13} \mathrm{H}_{21} \mathrm{ClNaO}(\mathrm{M}+$ $\mathrm{Na})^{+} 251.1179$, found $251.1136(\Delta-4.3 \mathrm{mmu})$.

Spiroketones 18a and 18b. To a solution of chloride $17(118 \mathrm{mg}, 0.52 \mathrm{mmol})$ in xylene (8 $\mathrm{mL})$ and 3-ethyl-3-pentanol $(0.44 \mathrm{~mL}, 3.1 \mathrm{mmol})$ was added a mixture of $\mathrm{NaH}(60 \%$ in mineral oil, $64 \mathrm{mg}, 1.6 \mathrm{mmol}$ ), and the mixture was quickly heated to reflux. After $30 \mathrm{~min}$, the mixture was cooled to room temperature. diluted with saturated aqueous $\mathrm{NH}_{4} \mathrm{Cl}(10 \mathrm{~mL})$, and extracted with hexane $(3 \times 20 \mathrm{~mL})$. The extract was washed with brine, dried $\left(\mathrm{Na}_{2} \mathrm{SO}_{4}\right)$, and concentrated. The residual xylene solution was chromatographed on silica gel ( $2 \mathrm{~g}$, hexaneether 150:1) to give spiro ketone $\mathbf{1 8 a}(79.0 \mathrm{mg}, 79 \%)$ and spiro ketone $\mathbf{1 8 b}(6.7 \mathrm{mg}, 7 \%)$ as a colorless oil, respectively.

18a: IR $\left(\mathrm{CHCl}_{3}\right) 3080,3005,2930,2860,1690,1640,1460,1440,1350,1315 \mathrm{~cm}^{-1} ;{ }^{1} \mathrm{H}$ NMR $\left(270 \mathrm{MHz}, \mathrm{CDCl}_{3}\right) \delta 5.55(\mathrm{dt}, J=17.0,9.7 \mathrm{~Hz}, 1 \mathrm{H}), 5.06(\mathrm{ddd}, J=17.0,2.0,0.7 \mathrm{~Hz}, 1 \mathrm{H})$, 4.99 (dd, $J=9.7,2.0 \mathrm{~Hz}, 1 \mathrm{H}), 2.60$ (m, $1 \mathrm{H}), 2.38-2.20$ (m, $3 \mathrm{H}), 1.95-1.05$ (m, $13 \mathrm{H})$; MS (GCEI) $m / z 192\left(\mathrm{M}^{+}, 35\right), 151$ (100); HRMS (FAB) $m / z$ calcd. for $\mathrm{C}_{13} \mathrm{H}_{20} \mathrm{NaO}(\mathrm{M}+\mathrm{Na})^{+}$ 215.1412, found $215.1451(\Delta+3.9 \mathrm{mmu})$.

18b: IR $\left(\mathrm{CHCl}_{3}\right)$ 3080, 3005, 2930, 2870, 1690, 1640, 1445, 1340, $1315 \mathrm{~cm}^{-1} ;{ }^{1} \mathrm{H}$ NMR (270 $\left.\mathrm{MHz}, \mathrm{CDCl}_{3}\right) \delta 5.77(\mathrm{~m}, 1 \mathrm{H}), 5.04(\mathrm{~m}, 2 \mathrm{H}), 2.72(\mathrm{br} \mathrm{q}, J=8.3 \mathrm{~Hz}, 1 \mathrm{H}), \quad 2.57-2.44(\mathrm{~m}, 2$ H), 2.19 (br dt, $J=12.9,7.4 \mathrm{~Hz}, 1 \mathrm{H}), 1.93-1.25$ (m, $13 \mathrm{H}) ;{ }^{13} \mathrm{C} \mathrm{NMR}\left(67.8 \mathrm{MHz}, \mathrm{CDCl}_{3}\right) \delta$ 217.5, 138.7, 115.6, 62.1, 52.1, 42.4, 36.0, 31.3, 31.1, 30.6, 26.4, 25.4, 22.8; HRMS (FAB) $m / z$ calcd. for $\mathrm{C}_{13} \mathrm{H}_{20} \mathrm{NaO}(\mathrm{M}+\mathrm{Na})^{+} 215.1412$, found $215.1454(\Delta+4.2 \mathrm{mmu})$.

Reduction of 18b. A mixture of alcohol $18 \mathrm{~b}(11.6 \mathrm{mg}, 0.060 \mathrm{mmol})$ and $\mathrm{NaBH}_{4}(3.0 \mathrm{mg}$, $0.079 \mathrm{mmol})$ in ethanol $(1 \mathrm{~mL})$ was stirred at room temperature for $32 \mathrm{~h}$. After the reaction 
was quenched by addition of acetone $(1 \mathrm{~mL})$, the mixture was concentrated, diluted with saturated aqueous $\mathrm{NH}_{4} \mathrm{Cl}$, and extracted with ether $(3 \times 5 \mathrm{~mL})$. The combined extracted were washed with brine, dried $\left(\mathrm{Na}_{2} \mathrm{SO}_{4}\right)$, and concentrated. The residue was chromatographed on silica gel (0.6 g, hexane-ether 20:1) to give alcohols 19a (5.8 mg, 50\%) and 19b (2.4 mg, $21 \%)$ as a colorless oil, respectively.

19a: IR (neat) 3464, 2930, 2864, 1456, 1046, 1002, $905 \mathrm{~cm}^{-1} ;{ }^{1} \mathrm{H}$ NMR $\left(270 \mathrm{MHz}, \mathrm{CDCl}_{3}\right) \delta$ 6.01 (ddd, $J=17.1,9.9,9.6 \mathrm{~Hz}, 1 \mathrm{H}$ ), 5.18 (dd, $J=17.1,2.1 \mathrm{~Hz}, 1 \mathrm{H}), 5.03$ (dd, $J=9.9,2.1$ $\mathrm{Hz}, 1 \mathrm{H}), 3.72$ (m, $1 \mathrm{H}), 2.42(\mathrm{ddd}, \mathrm{J}=9.6,9.5,9.5 \mathrm{~Hz}, 1 \mathrm{H}), 2.17$ (m, $1 \mathrm{H}), 1.92-1.27$ (m, 16 $\mathrm{H}$ ); HRMS (FAB) $m / z$ calcd. for $\mathrm{C}_{13} \mathrm{H}_{22} \mathrm{NaO}(\mathrm{M}+\mathrm{Na})^{+} 217.1568$, found $217.1556(\Delta-1.2$ $\mathrm{mmu})$.

19b: IR (neat) 3397, 2928, 2861, 1458, 1041, 1001, $909 \mathrm{~cm}^{-1} ;{ }^{1} \mathrm{H}$ NMR $\left(270 \mathrm{MHz}, \mathrm{CDCl}_{3}\right) \delta$ 5.78 (ddd, $J=17.0,10.3,8.6 \mathrm{~Hz}, 1 \mathrm{H}$ ), $5.06-4.99$ (m, $2 \mathrm{H}), 3.50($ br d, $J=10.0 \mathrm{~Hz}, 1 \mathrm{H}$ ), 2.64 (ddd, $J=8.6,8.6,8.6 \mathrm{~Hz}, 1 \mathrm{H}$ ), 1.85-1.20 (m, $17 \mathrm{H}$ ); HRMS (FAB) $\mathrm{m} / z$ calcd. for $\mathrm{C}_{13} \mathrm{H}_{22} \mathrm{NaO}(\mathrm{M}+\mathrm{Na})^{+} 217.1568$, found 217.1556 ( $\left.\Delta-1.2 \mathrm{mmu}\right)$.

Allyl ketones 4 and 20. To a mixture of $0.5 \mathrm{M}$ solution of $\mathrm{KN}(\mathrm{TMS})_{2}$ in toluene $(0.97 \mathrm{~mL}$, $0.49 \mathrm{mmol})$ and THF $(1.1 \mathrm{~mL})$ was added spiro ketone $18 \mathbf{a}(62.3 \mathrm{mg}, 0.324 \mathrm{mmol})$ in THF $(0.2+0.1 \mathrm{~mL})$ at $-78{ }^{\circ} \mathrm{C}$. After the mixture was stirred at $-78{ }^{\circ} \mathrm{C}$ for $1 \mathrm{~h}$, allyl iodide $(0.05 \mathrm{~mL}$, $0.545 \mathrm{mmol}$ ) was added. The reaction mixture was stirred at $0{ }^{\circ} \mathrm{C}$ for $3 \mathrm{~h}$ and diluted with water $(2 \mathrm{~mL})$. The mixture was extracted with ether $(3 \times 5 \mathrm{~mL})$. The combined extracts were washed with brine, dried, and concentrated. The residue was chromatographed on silica gel (40 g, hexane-ether 150:1 to 25:1) to give a mixture of allyl ketones $(60.8 \mathrm{mg}, \mathbf{4 : 2 0}=10: 1$, $81 \%$ ) as a colorless oil. This mixture can be separated by column chromatography on silica gel (Fuji silysia FL-60D 3 g, hexane-benzene 10:1 to 2:1).

4: IR (neat) 3075, 2928, 2856, 1695, 1639, 1454, $1372 \mathrm{~cm}^{-1} ;{ }^{1} \mathrm{H}$ NMR (270 MHz, $\left.\mathrm{CDCl}_{3}\right) \delta$ $5.72(\mathrm{~m}, 1 \mathrm{H}), 5.54(\mathrm{dt}, J=17.0,9.6 \mathrm{~Hz}, 1 \mathrm{H}), 5.10-4.96(\mathrm{~m}, 4 \mathrm{H}), 2.68(\mathrm{~m}, 1 \mathrm{H}), 2.43-2.20$ $(\mathrm{m}, 3 \mathrm{H}), 2.09-1.26(\mathrm{~m}, 12 \mathrm{H}), 1.10-1.00(\mathrm{~m}, 2 \mathrm{H}),{ }^{13} \mathrm{C} \mathrm{NMR}\left(67.8 \mathrm{MHz}, \mathrm{CDCl}_{3}\right) \delta$ 218.7, 138.6, 137.1, 116.4, 115.9, 63.0, 58.0, 50.3, 37.3, 35.7, 35.0, 32.3, 31.9, 29.9, 25.8, 23.6; HRMS (FAB) $m / z$ calcd. for $\mathrm{C}_{16} \mathrm{H}_{24} \mathrm{NaO}(\mathrm{M}+\mathrm{Na})^{+} 255.1725$, found $255.1755(\Delta+3.0 \mathrm{mmu})$. 20: IR $\left(\mathrm{CHCl}_{3}\right)$ 3080, 2930, 2850, 1695, 1640, 1455, $1370 \mathrm{~cm}^{-1} ;{ }^{1} \mathrm{H}$ NMR $(270 \mathrm{MHz}$, $\left.\mathrm{CDCl}_{3}\right) \delta 6.24(\mathrm{ddd}, J=17.2,10.1,9.1 \mathrm{~Hz}, 1 \mathrm{H}), 5.68(\mathrm{~m}, 1 \mathrm{H}), 5.05-4.89(\mathrm{~m}, 4 \mathrm{H}), 2.48(\mathrm{~m}$, $1 \mathrm{H}), 2.23(\mathrm{~m}, 2 \mathrm{H}), 2.03-1.18(\mathrm{~m}, 14 \mathrm{H})$; HRMS (FAB) $m / z$ calcd. for $\mathrm{C}_{16} \mathrm{H}_{24} \mathrm{NaO}(\mathrm{M}+\mathrm{Na})^{+}$ 255.1725 , found $255.1729(\Delta+0.4 \mathrm{mmu})$. 
Tricyclic ketone 5. A solution of allyl spiro ketone $4(10.3 \mathrm{mg}, 0.0443 \mathrm{mmol})$ and the first generation Grubbs catalyst, $\mathrm{RuCl}_{2}(=\mathrm{CHPh})\left(\mathrm{PCy}_{3}\right)_{2}(3.6 \mathrm{mg}, 4.4 \mu \mathrm{mol})$ in toluene $(13 \mathrm{~mL})$ was refluxed for $3.5 \mathrm{~h}$. The catalyst ( $5 \mathrm{mg}, 6 \mu \mathrm{mol})$ was added, and the mixture was refluxed for further $2 \mathrm{~h}$. The mixture was concentrated to give a oil, which was chromatographed on silica gel (3 g, hexane-ether 100:1 to 10:1) to give tricyclic ketone $\mathbf{5}(2.4 \mathrm{mg}, 27 \%)$ as a colorless oil. IR $\left(\mathrm{CHCl}_{3}\right)$ 3000, 2945, 2875, 1720, 1450, $1380 \mathrm{~cm}^{-1} ;{ }^{1} \mathrm{H} \mathrm{NMR}\left(270 \mathrm{MHz}, \mathrm{CDCl}_{3}\right) \delta 5.48$ (dq, $J=12.4,3.1 \mathrm{~Hz}, 1 \mathrm{H}), 5.27(\mathrm{dq}, J=12.4,2.1 \mathrm{~Hz}, 1 \mathrm{H}), 3.53(\mathrm{~m}, 1 \mathrm{H}), 2.91(\mathrm{~m}, 1 \mathrm{H})$, 2.34-2.02 (m, $4 \mathrm{H}), 1.80-0.84(\mathrm{~m}, 12 \mathrm{H}) ;{ }^{13} \mathrm{C} \mathrm{NMR}\left(67.8 \mathrm{MHz}, \mathrm{CDCl}_{3}\right) \delta$ 215.5, 135.0, 128.9, 65.0, 49.5, 49.4, 42.4, 36.1, 32.9, 31.9, 29.9, 28.2, 25.7, 25.2; MS (GCEI) m/z $204\left(\mathrm{M}^{+}, 100\right)$, $186\left([\mathrm{M}-18]^{+}, 21\right)$; HRMS (FAB) $m / z$ calcd. for $\mathrm{C}_{14} \mathrm{H}_{20} \mathrm{NaO}(\mathrm{M}+\mathrm{Na})^{+} 227.1412$, found 227.1407 ( $\Delta-0.5 \mathrm{mmu})$.

Tricyclic ketone 21. Allyl spiro ketone $20(8.5 \mathrm{mg}, 0.037 \mathrm{mmol})$ was cyclized with first generation Grubbs catalyst, $\mathrm{RuCl}_{2}(=\mathrm{CHPh})\left(\mathrm{PCy}_{3}\right)_{2}(3.0 \mathrm{mg}, 3.6 \mu \mathrm{mol})$ in boiling toluene $(9$ $\mathrm{mL}$ ) for $1.5 \mathrm{~h}$ by the same procedure in the preparation of 5 to give tricyclic ketone 21 (4.6 $\mathrm{mg}, 62 \%)$ as a colorless oil. IR (neat) 2926, 2860, 1694, 1448, $1150 \mathrm{~cm}^{-1} ;{ }^{1} \mathrm{H}$ NMR $(270 \mathrm{MHz}$, $\mathrm{CDCl}_{3}$ ) $\delta 5.67$ (dddd, $J=10.8,9.0,5.0,3.2 \mathrm{~Hz}, 1 \mathrm{H}$ ), 5.45 (dt, $\left.J=10.8,2.5 \mathrm{~Hz}, 1 \mathrm{H}\right), 2.86$ (m, $1 \mathrm{H}), 2.74(\mathrm{~m}, 1 \mathrm{H}), 2.59$ (m, $1 \mathrm{H}), 2.38$ (dt, $J=12.4,9.4 \mathrm{~Hz}, 1 \mathrm{H}), 2.13(\mathrm{dt}, J=15.4,6.6 \mathrm{~Hz}$, $1 \mathrm{H}), 2.04-1.92(\mathrm{~m}, 2 \mathrm{H}), 1.85-1.20(\mathrm{~m}, 11 \mathrm{H}) ;{ }^{13} \mathrm{C} \mathrm{NMR}\left(67.8 \mathrm{MHz}, \mathrm{CDCl}_{3}\right) \delta 215.9,134.8$, 127.9, 62.4, 55.0, 47.6, 39.7, 35.6, 34.3, 30.9, 29.0, 26.6, 25.9, 22.6; HRMS (FAB) $\mathrm{m} / \mathrm{z}$ calcd. for $\mathrm{C}_{14} \mathrm{H}_{20} \mathrm{NaO}(\mathrm{M}+\mathrm{Na})^{+} 227.1412$, found $227.1375(\Delta-3.7 \mathrm{mmu})$.

Tricyclic ketone 22a [107454-52-4]. The mixture of tricyclic ketone 5 (1.8 mg, $8.8 \mu \mathrm{mol})$, $\mathrm{Pd} / \mathrm{C}(10 \%, 1 \mathrm{mg})$, and ethanol $(0.5 \mathrm{~mL})$ was stirred at room temperature for $7.5 \mathrm{~h}$ under hydrogen. The mixture was filtered through a pad of Celite, and the residue was washed with ether. The filtrate and washings were combined and concentrated to give an oil, which was hydrogenated with $\mathrm{Pd} / \mathrm{C}(10 \%, 1 \mathrm{mg})$ for further $14.5 \mathrm{~h}$ to give tricyclic ketone $22 \mathrm{a}(1.0 \mathrm{mg}$, $55 \%)$ as a colorless oil. IR $\left(\mathrm{CHCl}_{3}\right) 2945,2860,1720,1450,1380 \mathrm{~cm}^{-1} ;{ }^{1} \mathrm{H} \mathrm{NMR}(270 \mathrm{MHz}$, $\left.\mathrm{CDCl}_{3}\right) \delta 2.88(\mathrm{brtt}, J=11.9,2.0 \mathrm{~Hz}, 1 \mathrm{H}), 2.04-0.86(\mathrm{~m}, 21 \mathrm{H}) ;{ }^{13} \mathrm{C} \mathrm{NMR}\left(100 \mathrm{MHz}, \mathrm{CDCl}_{3-}\right.$ ) $\delta 217.0,63.5,54.3,50.3,41.1,36.2,35.0,30.8,30.6,30.54,30.51,30.48,26.0,25.2$; MS (GCEI) $m / z, 206\left(\mathrm{M}^{+}, 100\right), 188\left([\mathrm{M}-18]^{+}, 21\right)$.

Tricyclic ketone 22b. Tricyclic ketone 21 (2.6 mg, $0.013 \mathrm{mmol})$ was hydrogenated with $10 \% \mathrm{Pd} / \mathrm{C}(1 \mathrm{mg})$ by the same procedure in the preparation of $\mathbf{2 2} \mathbf{a}$ to give tricyclic ketone $\mathbf{2 2} \mathbf{b}$ 
$(1.8 \mathrm{mg}, 69 \%)$ as colorless oil. IR $\left(\mathrm{CHCl}_{3}\right) 2930,2860,1680,1460,1360 \mathrm{~cm}^{-1} ;{ }^{1} \mathrm{H} \mathrm{NMR}$ $\left(270 \mathrm{MHz}, \mathrm{CDCl}_{3}\right) \delta 2.73(\mathrm{~m}, 1 \mathrm{H}), 2.19-2.12(\mathrm{~m}, 2 \mathrm{H}), 1.95-1.17(\mathrm{~m}, 19 \mathrm{H}) ;{ }^{13} \mathrm{C} \mathrm{NMR}$ $\left(67.8 \mathrm{MHz}, \mathrm{CDCl}_{3}\right) \delta$ 219.2, 63.4, 54.7, 43.3, 38.8, 35.0, 34.1, 34.0, 30.0, 28.7, 26.6, 26.3, 24.7, 20.9; EIMS $m / z 206\left(\mathrm{M}^{+}, 100\right), 188$ (31); HREIMS $m / z$ calcd for $\mathrm{C}_{14} \mathrm{H}_{22} \mathrm{O}\left(\mathrm{M}^{+}\right)$206.1671, found $206.1643(\Delta-2.8 \mathrm{mmu})$. 


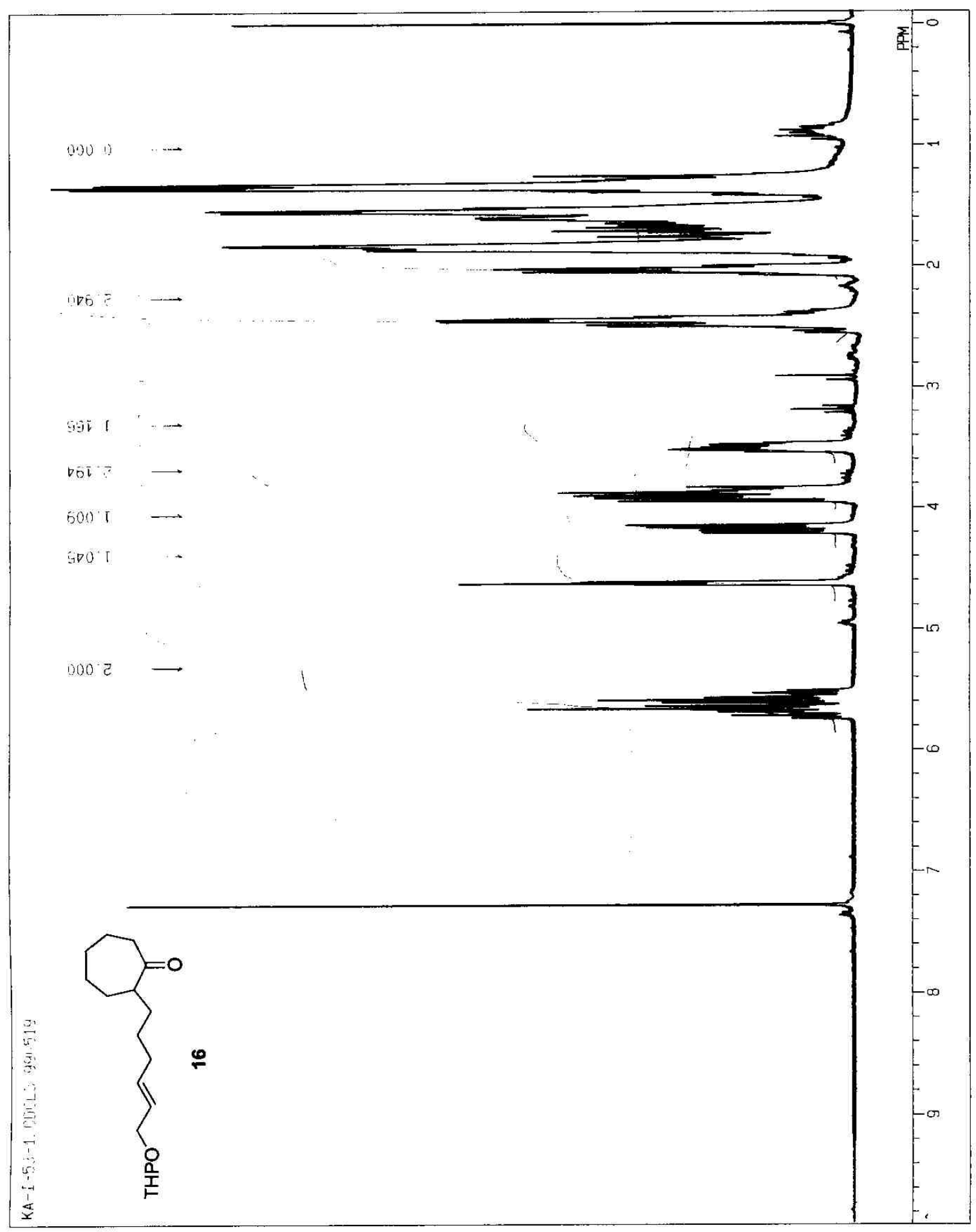




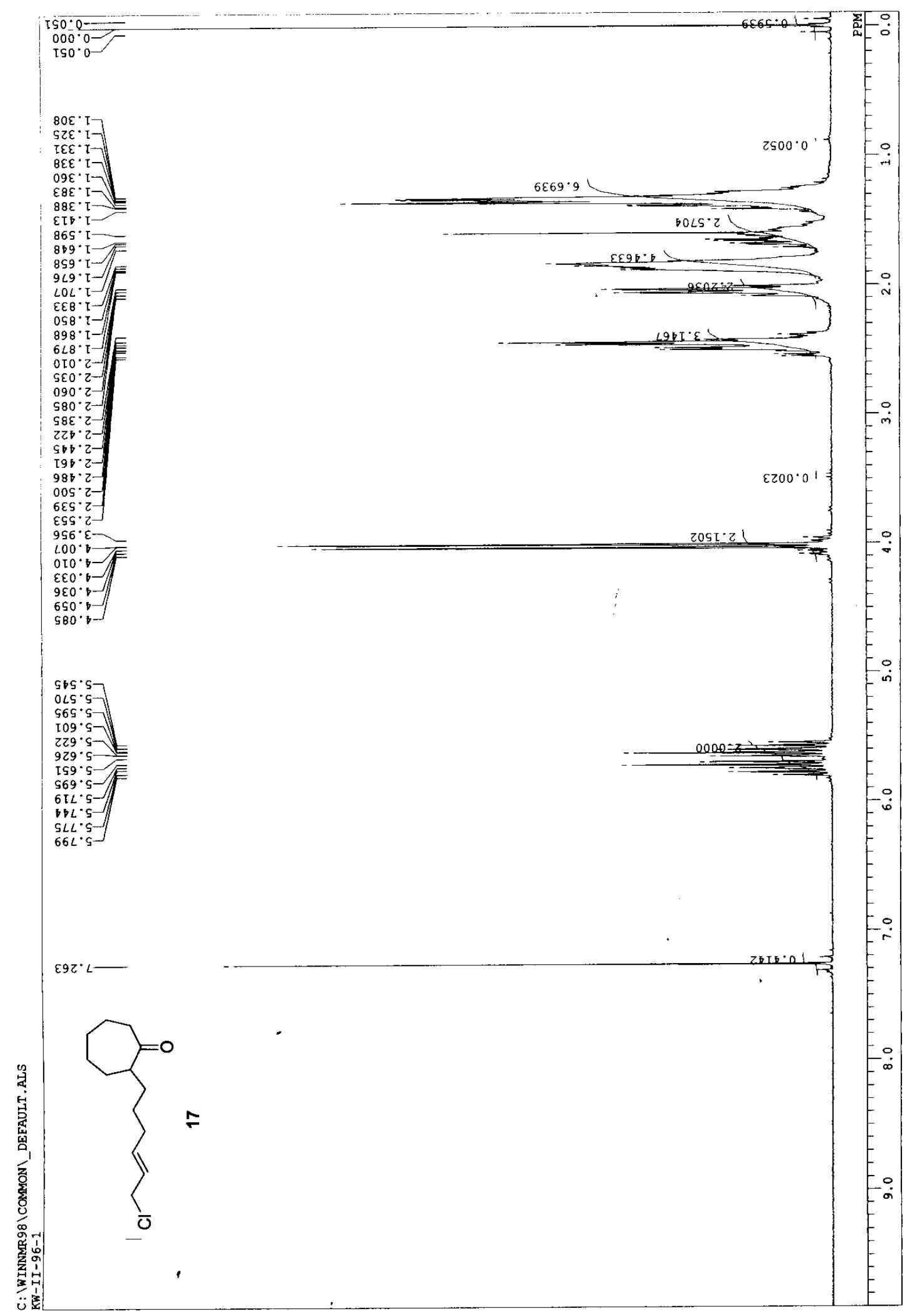




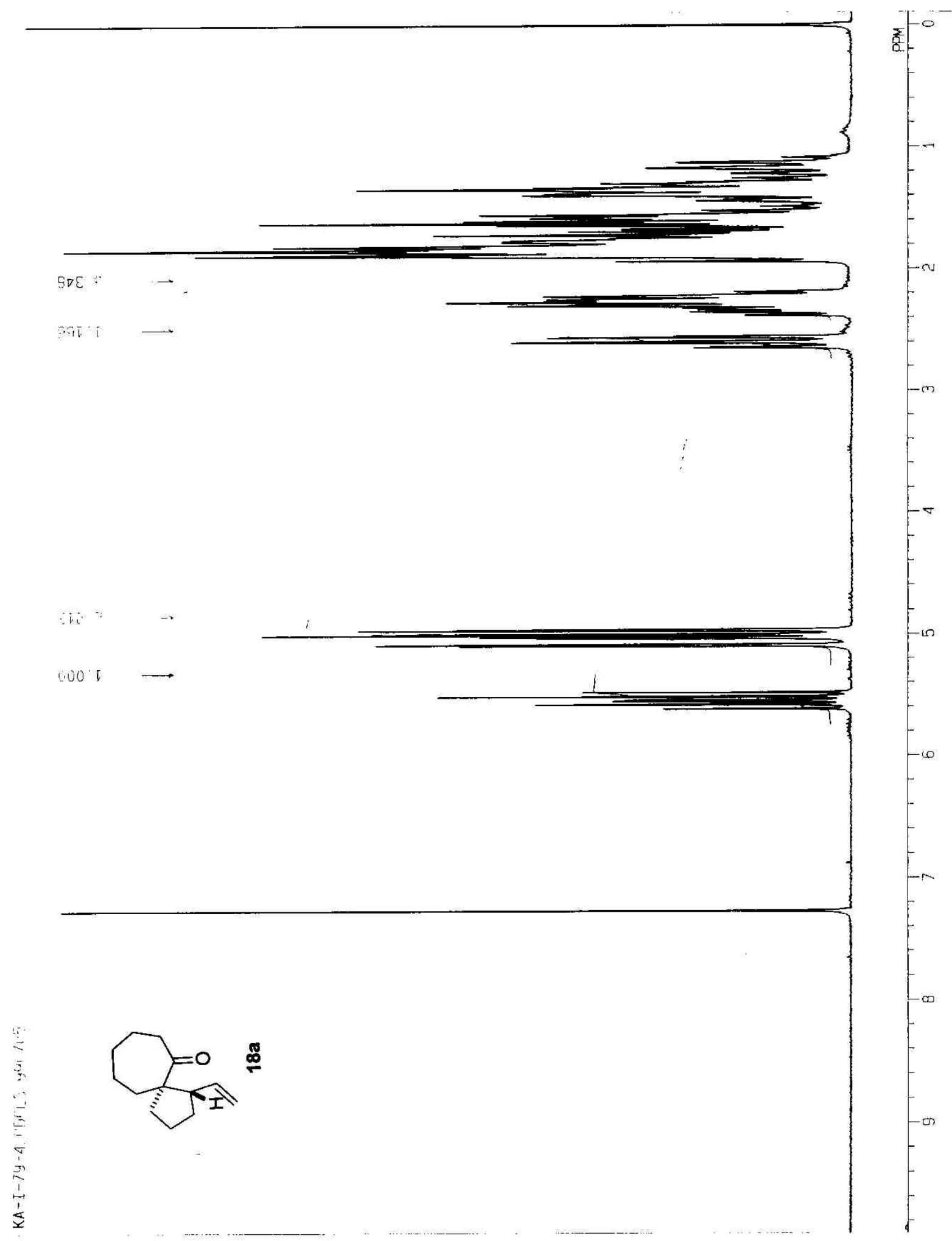




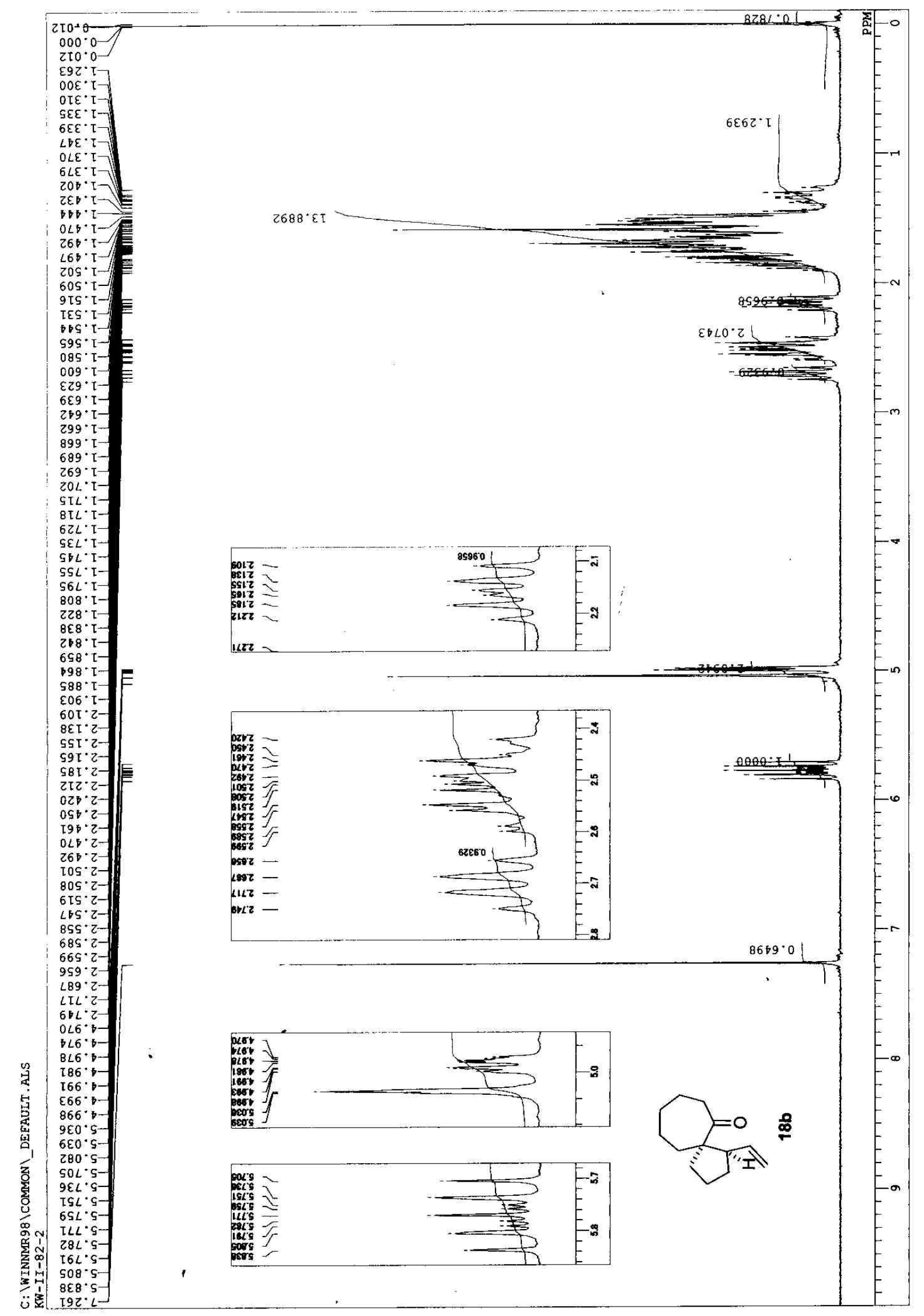




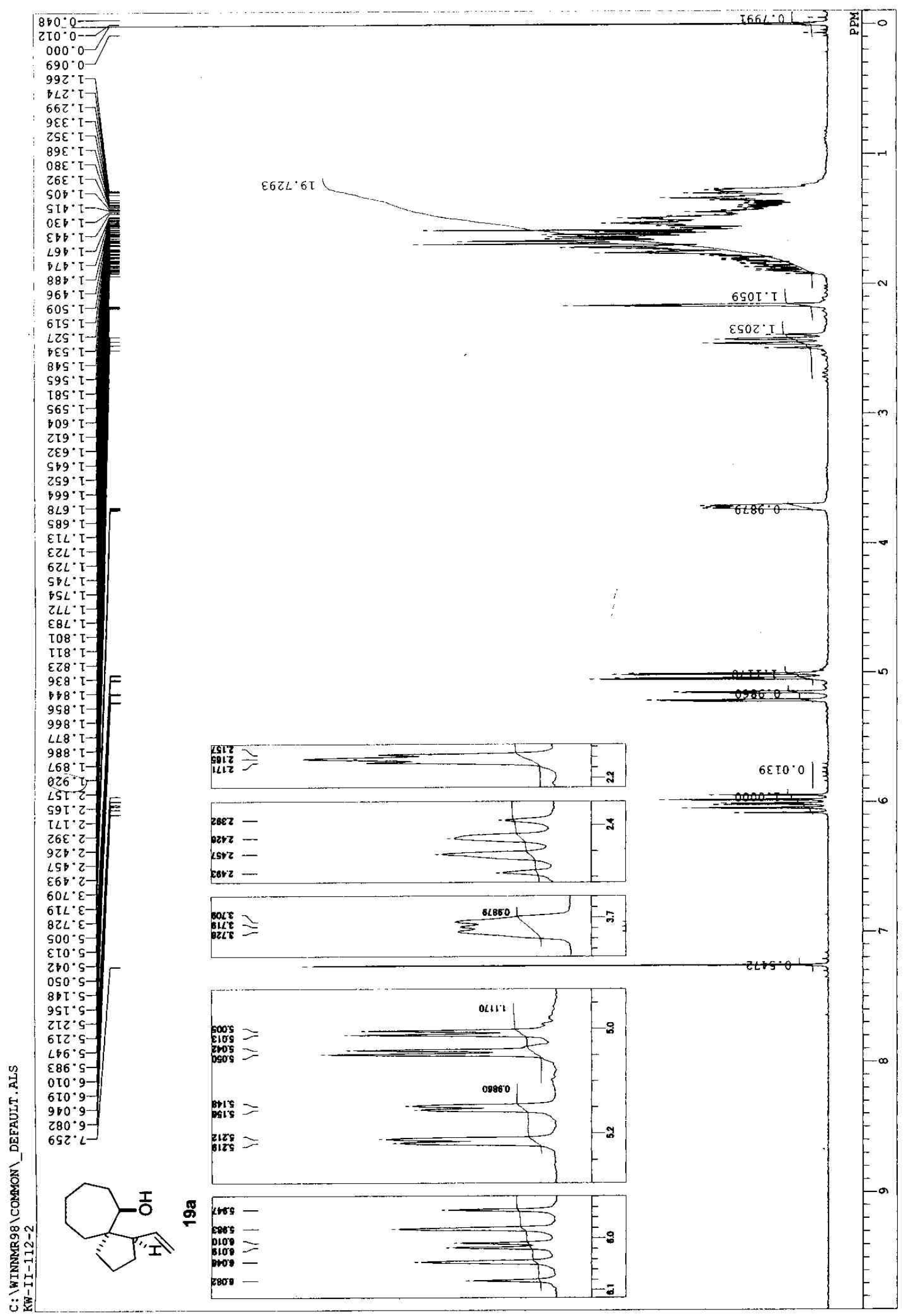




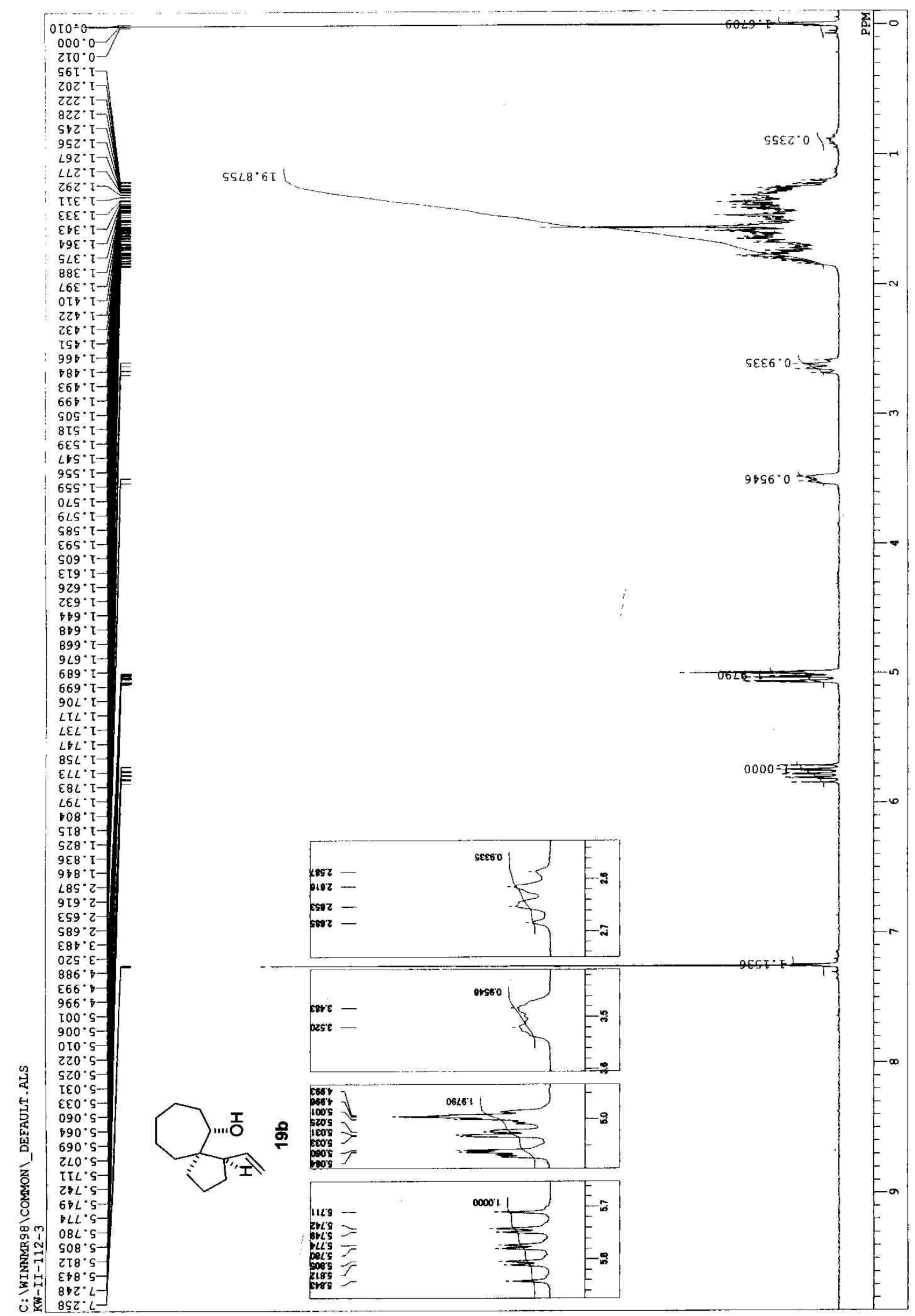




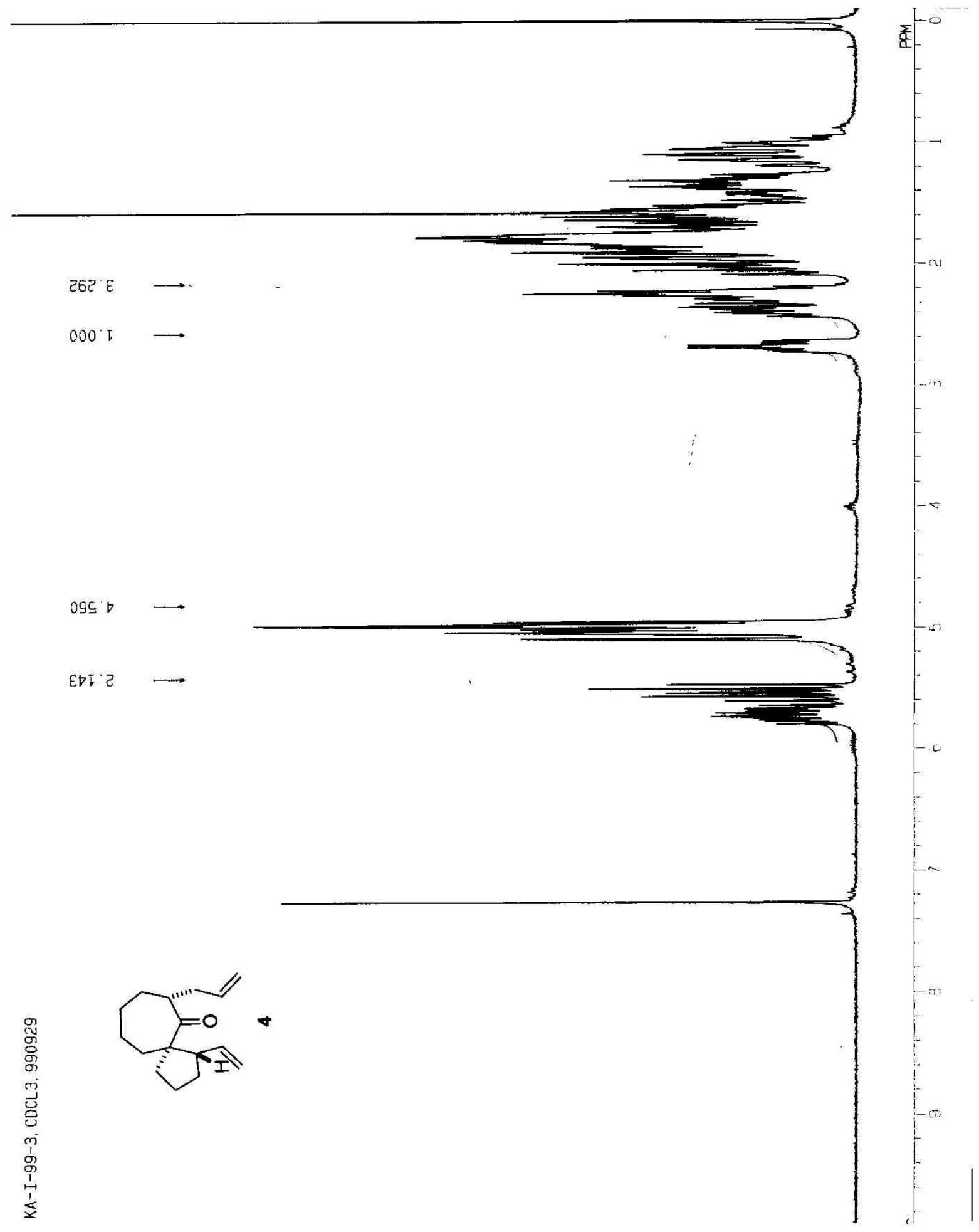




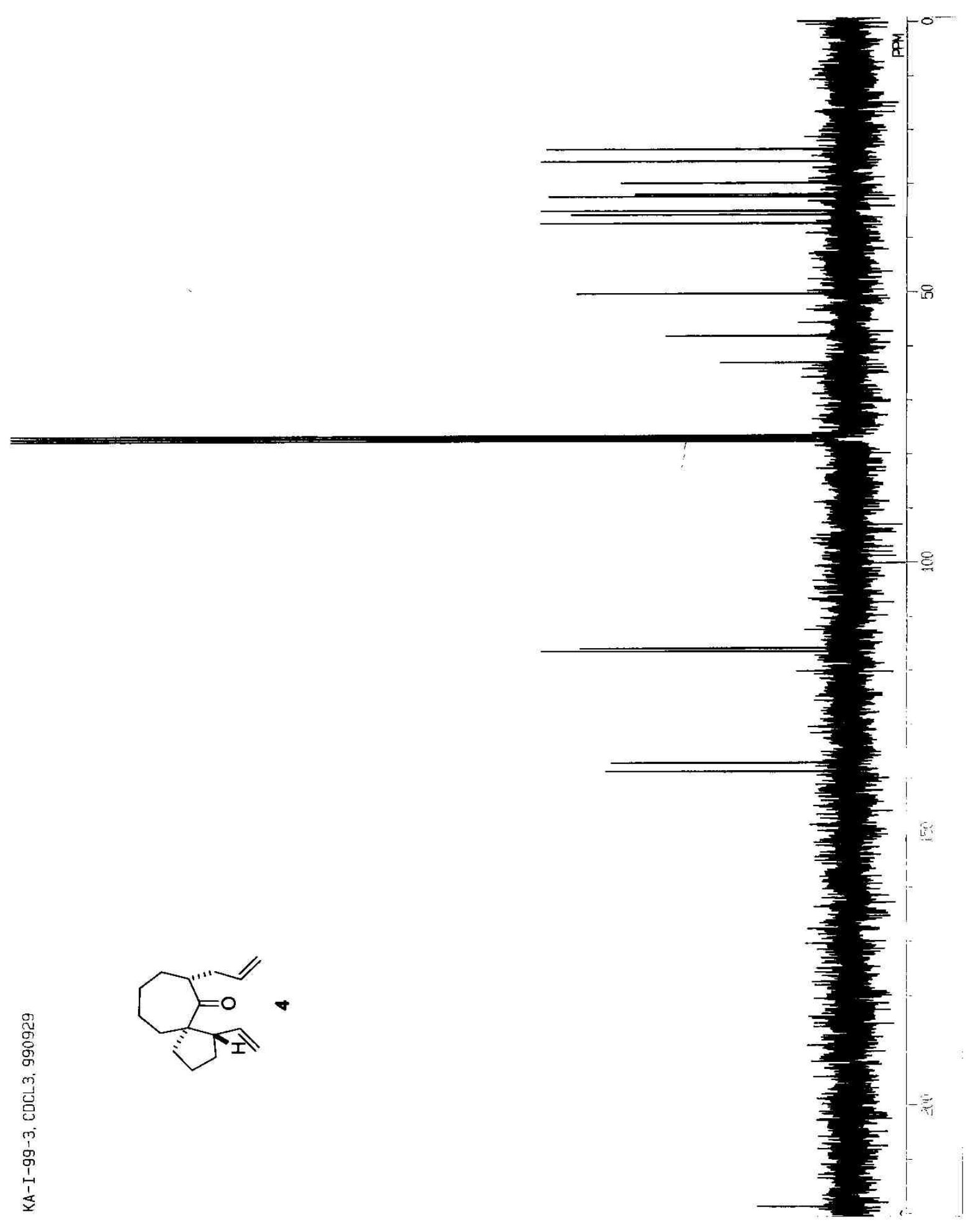




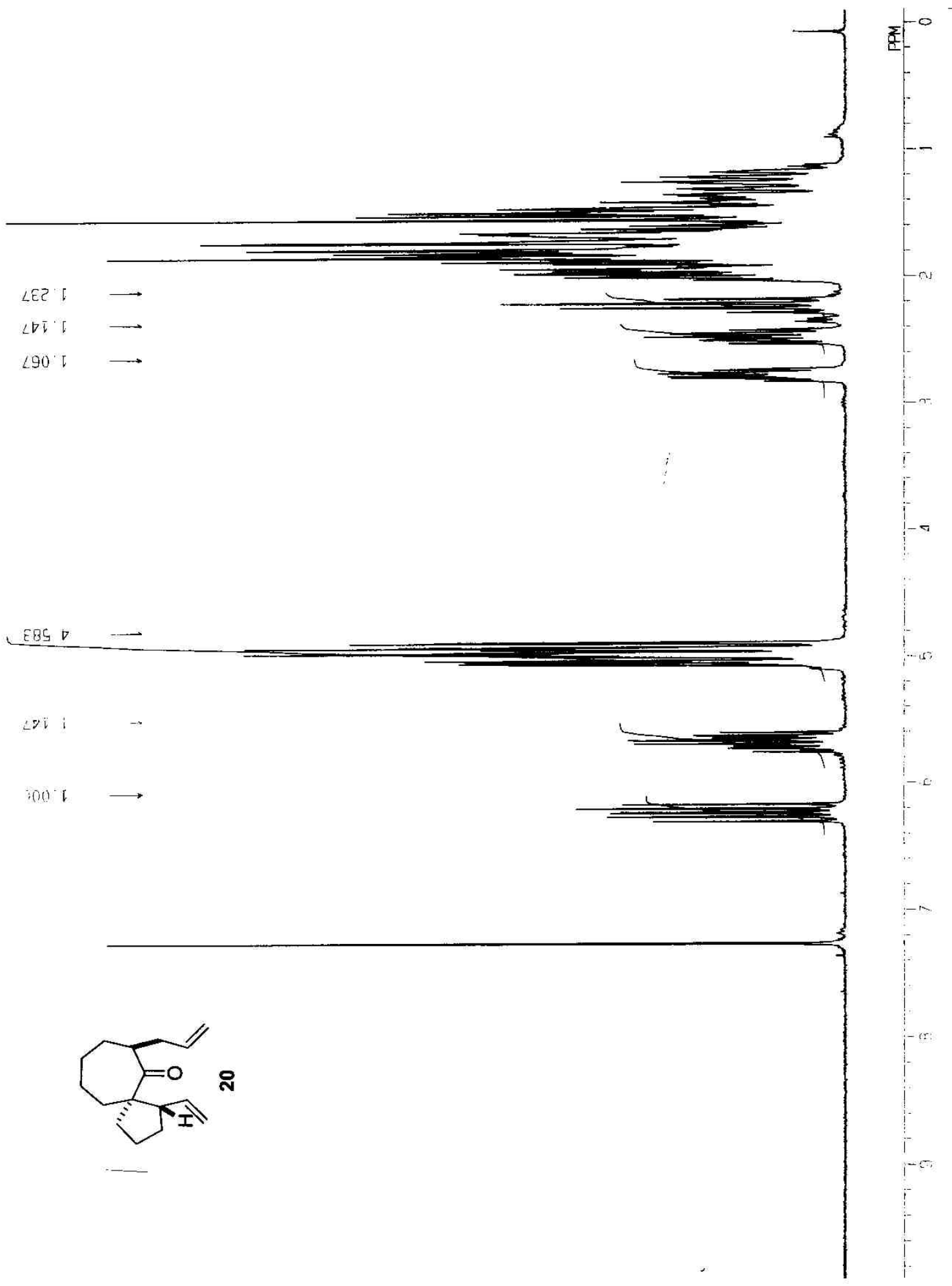




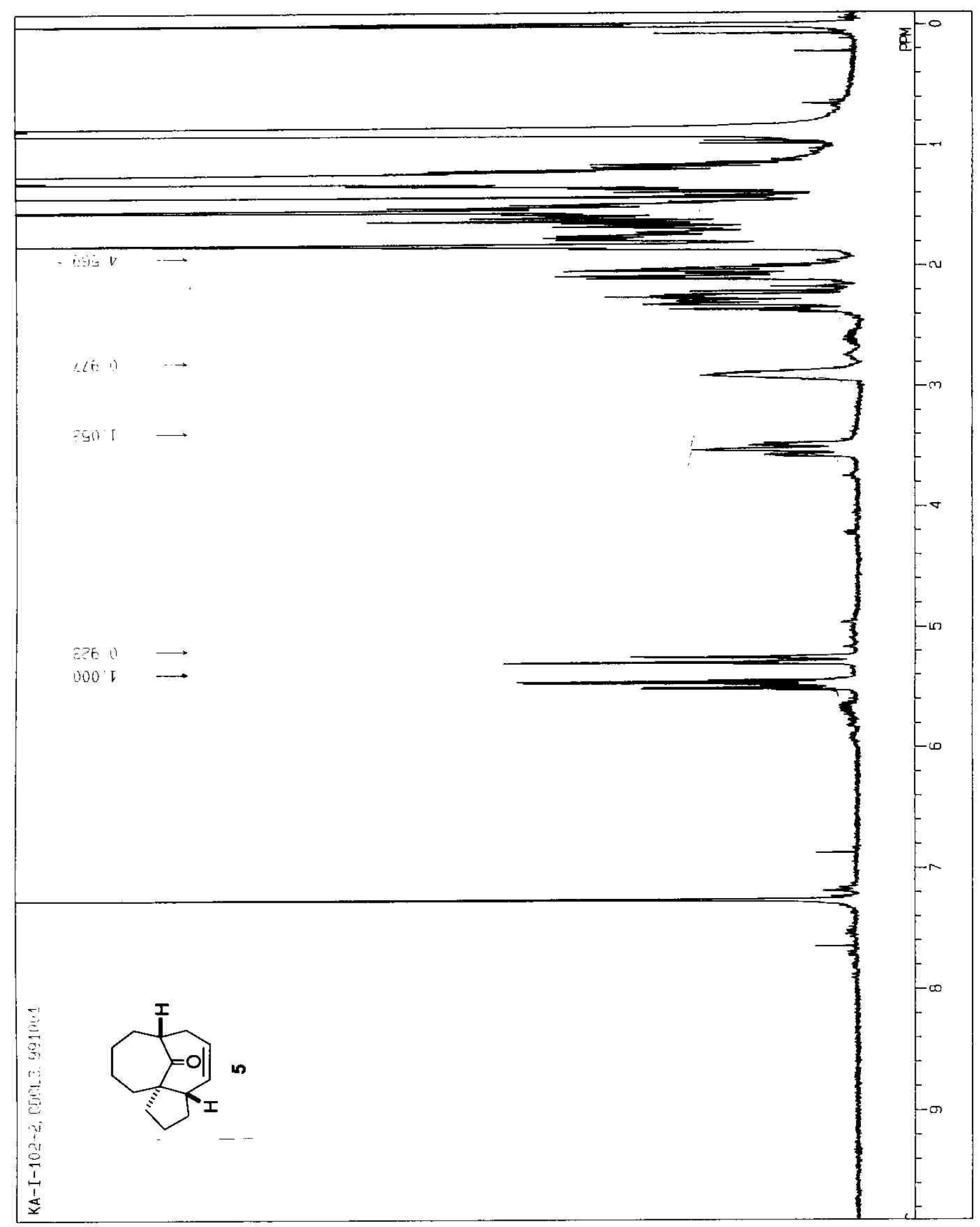




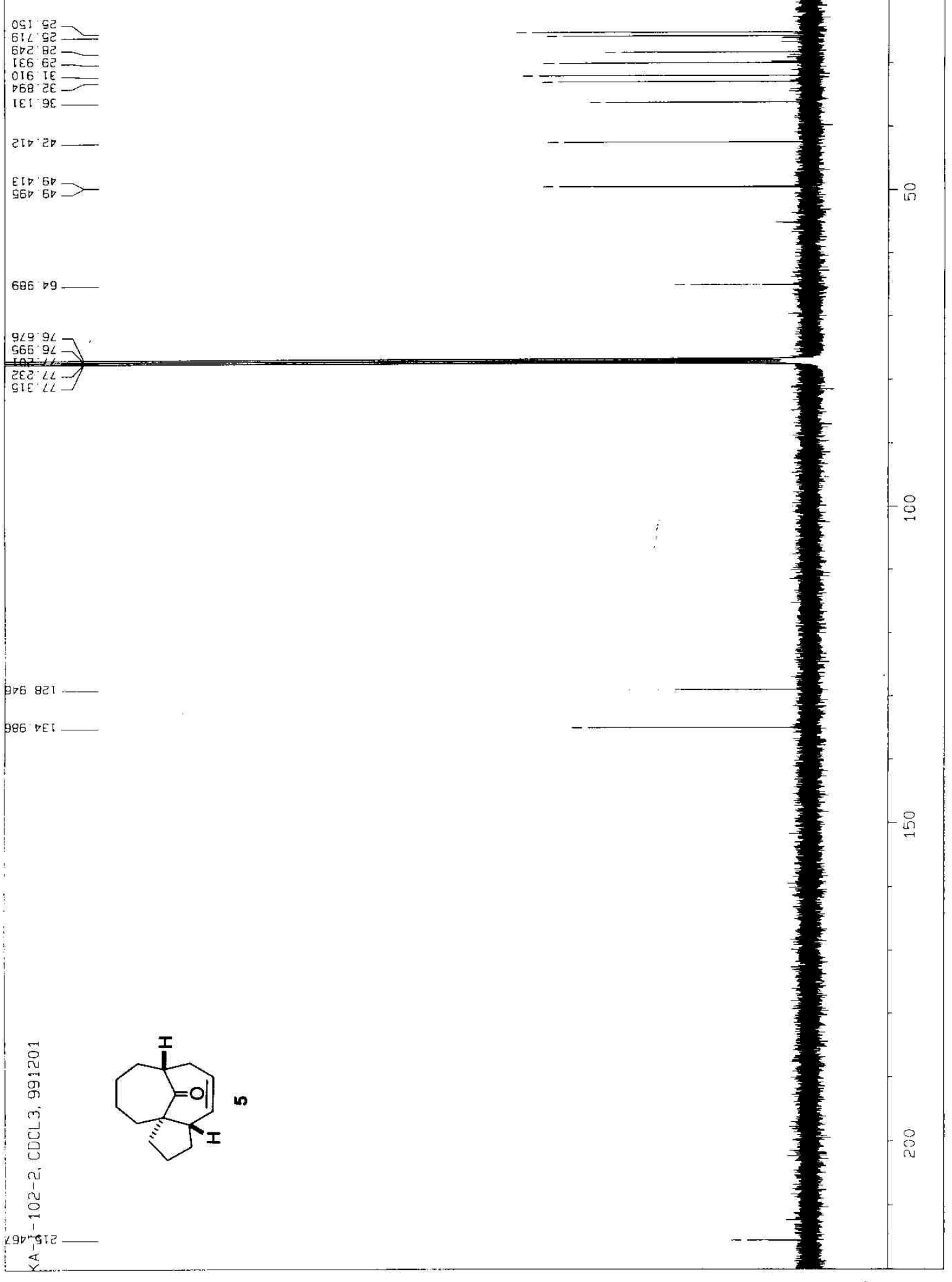




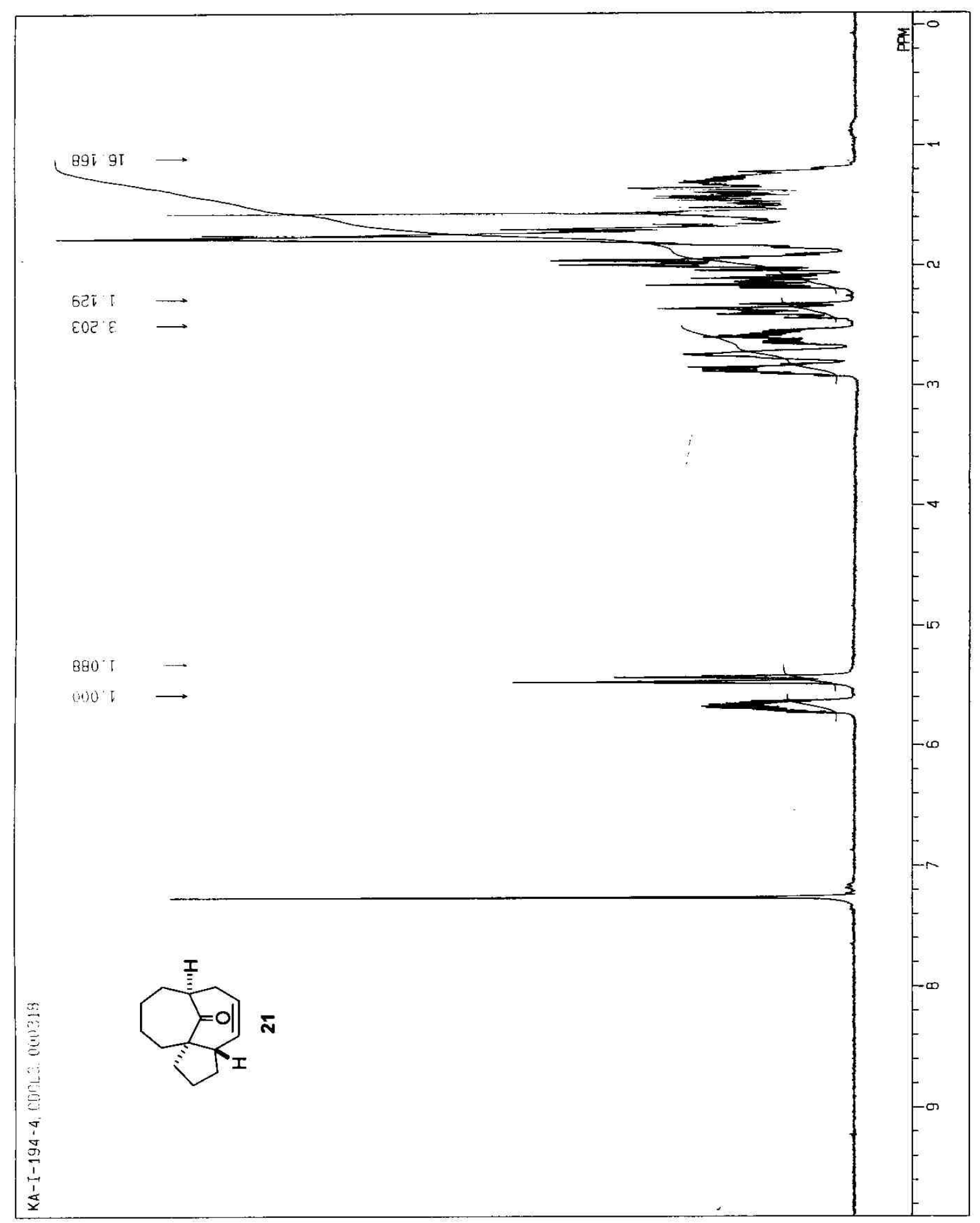




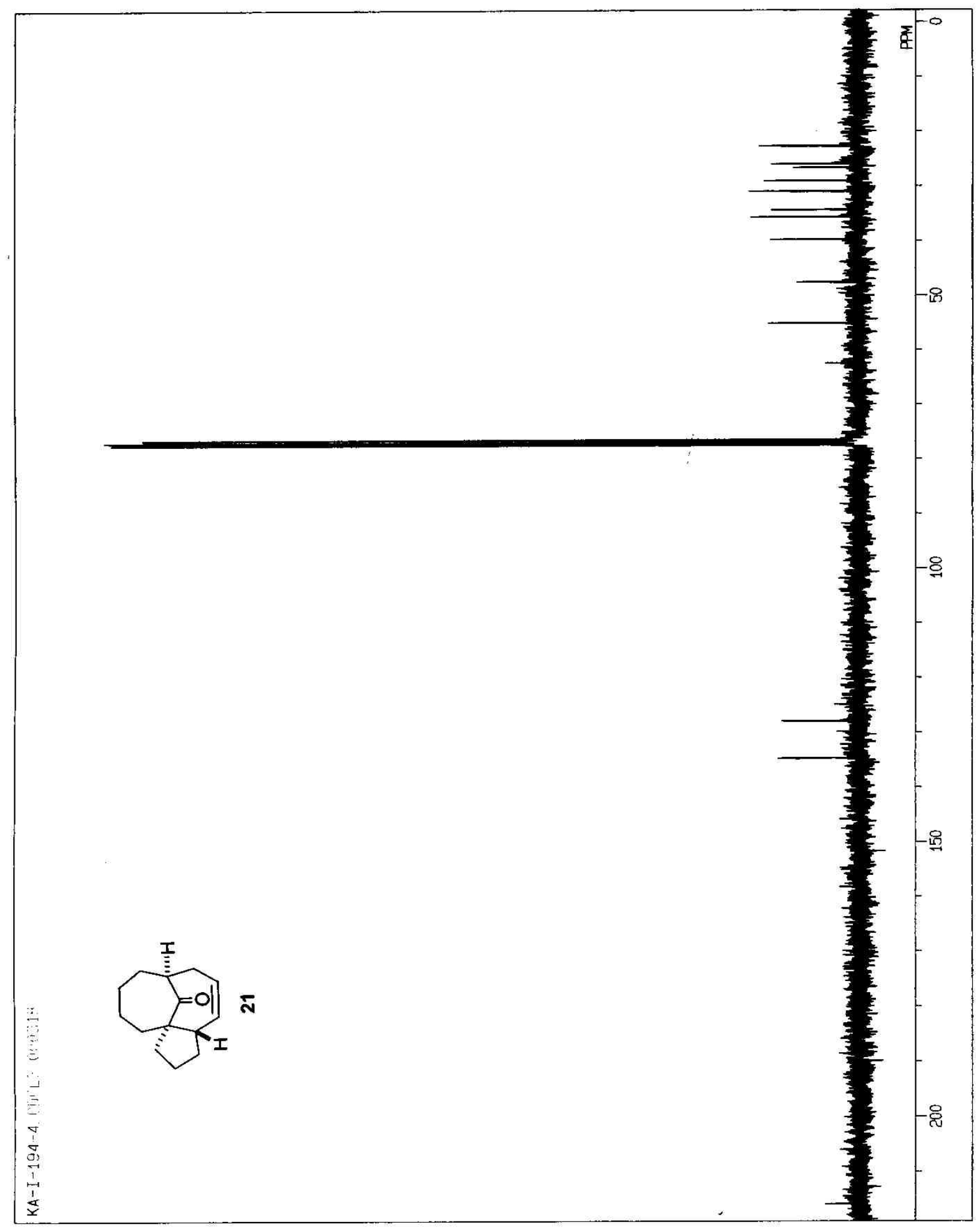




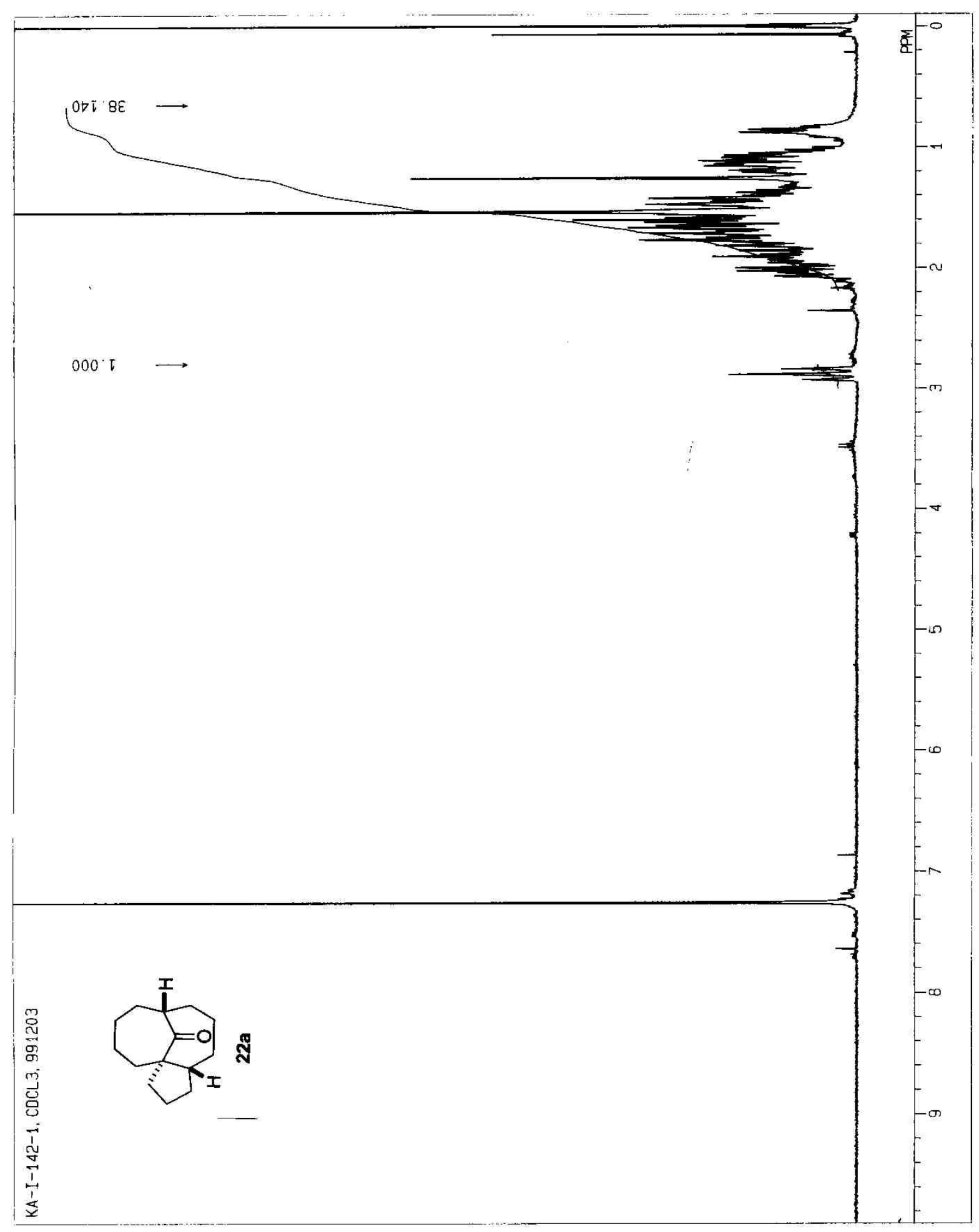




$$
1
$$




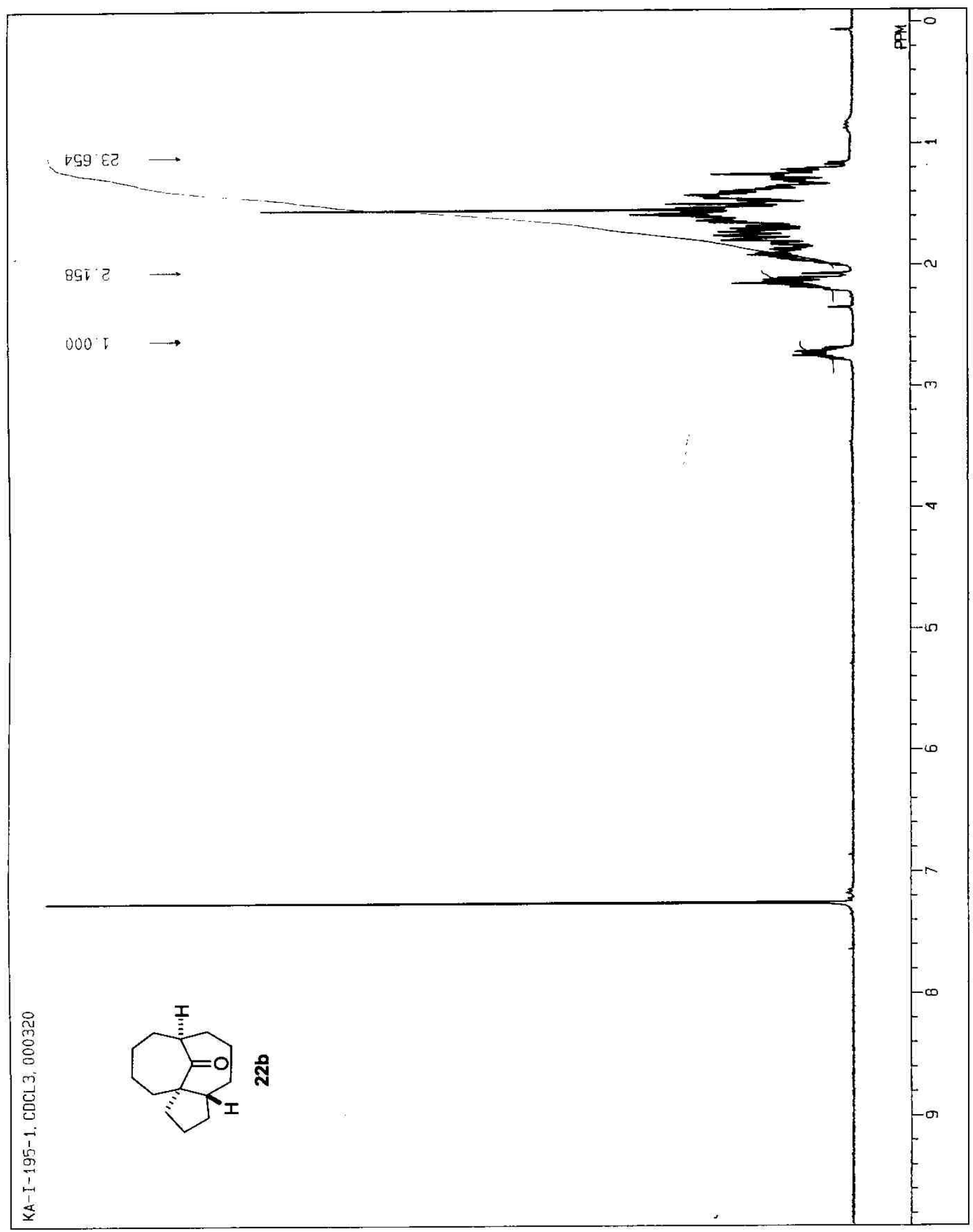




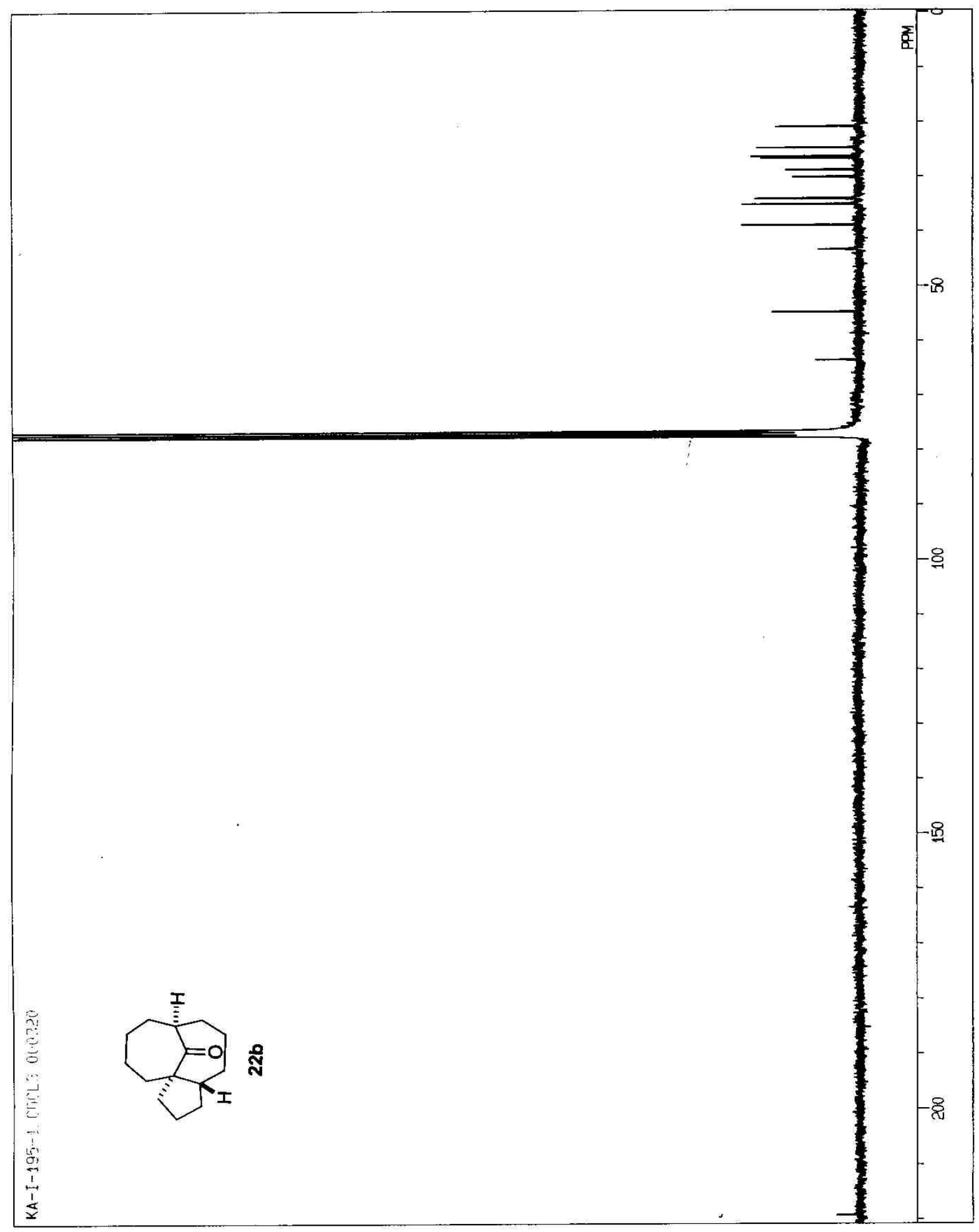




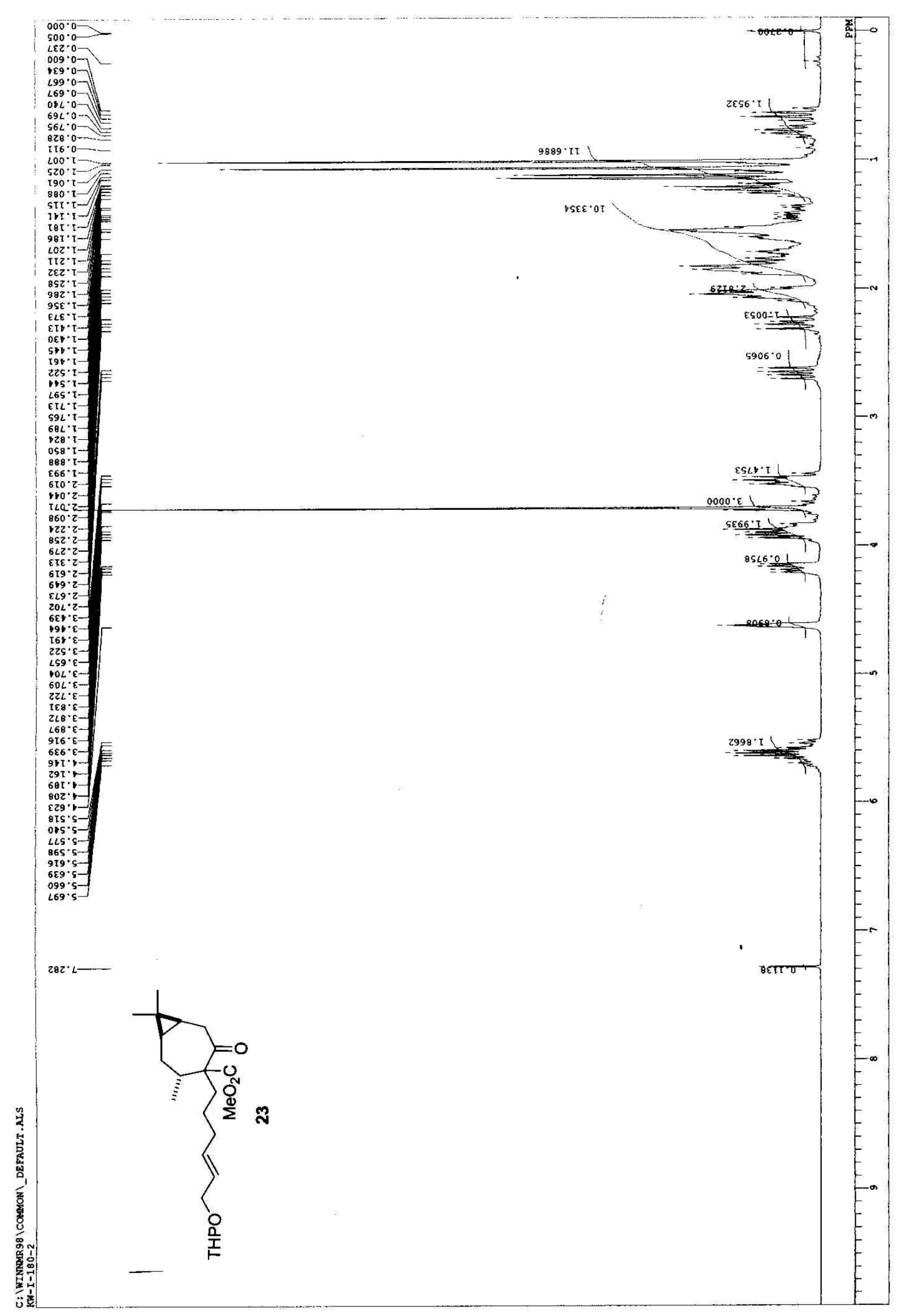




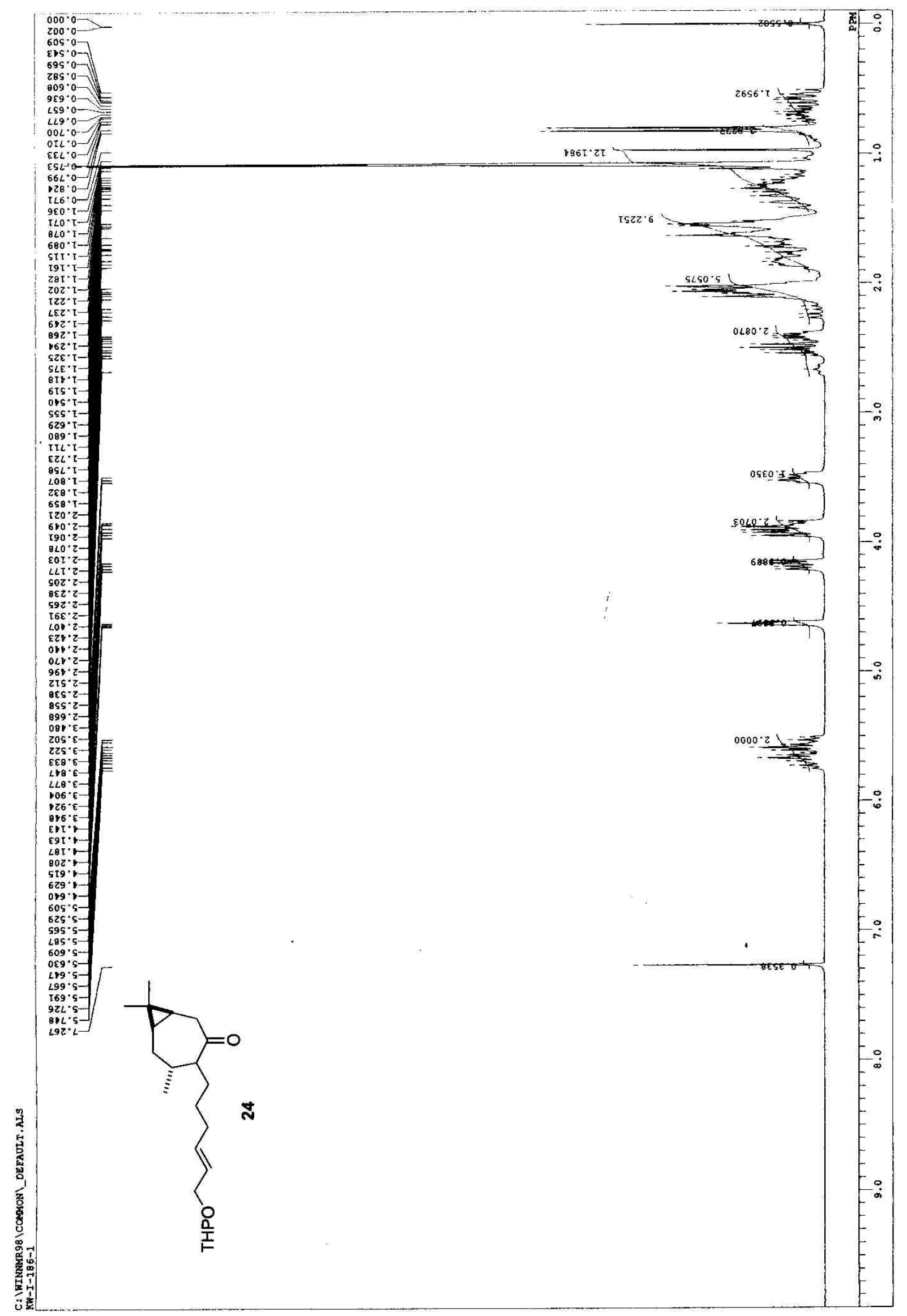




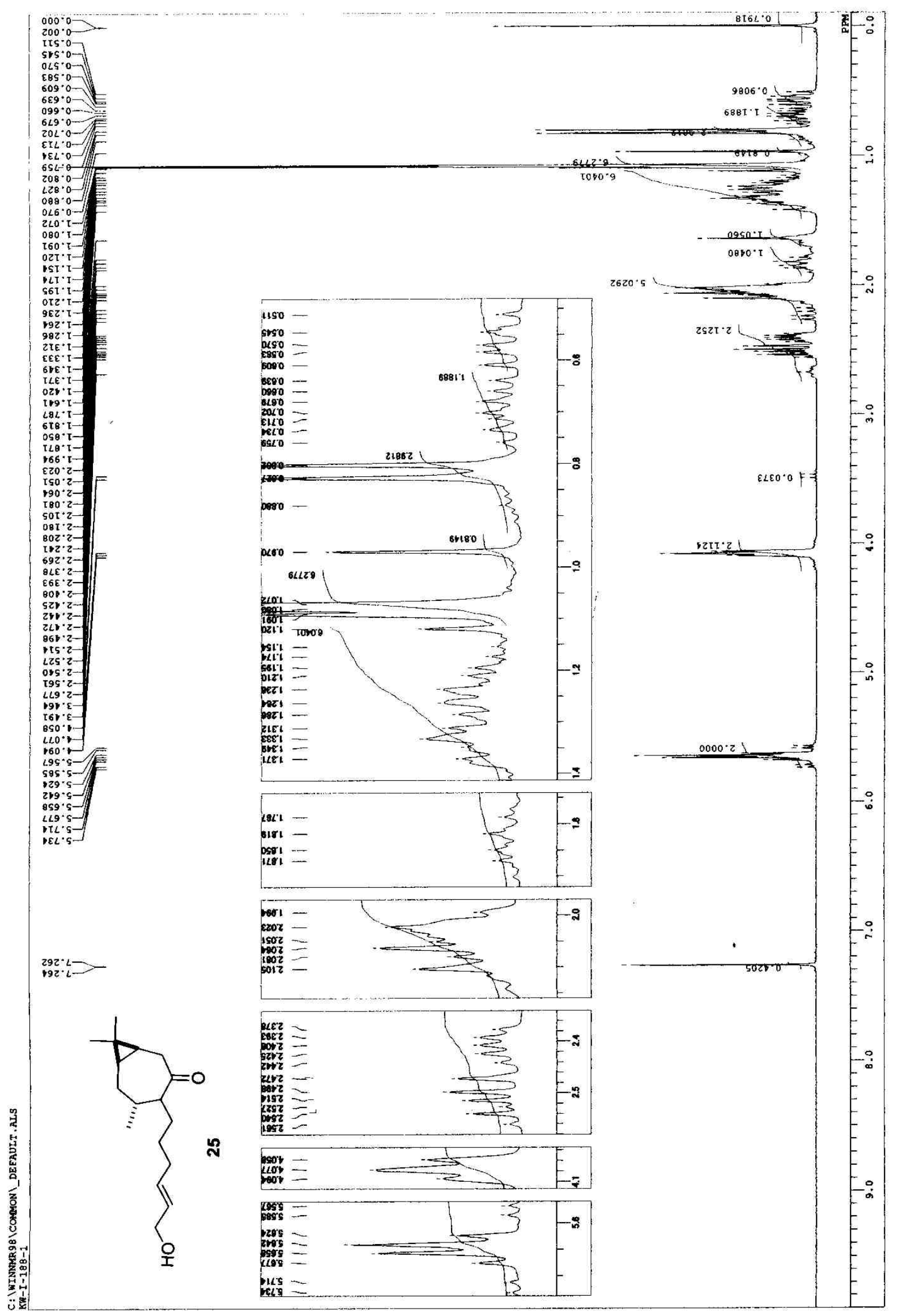




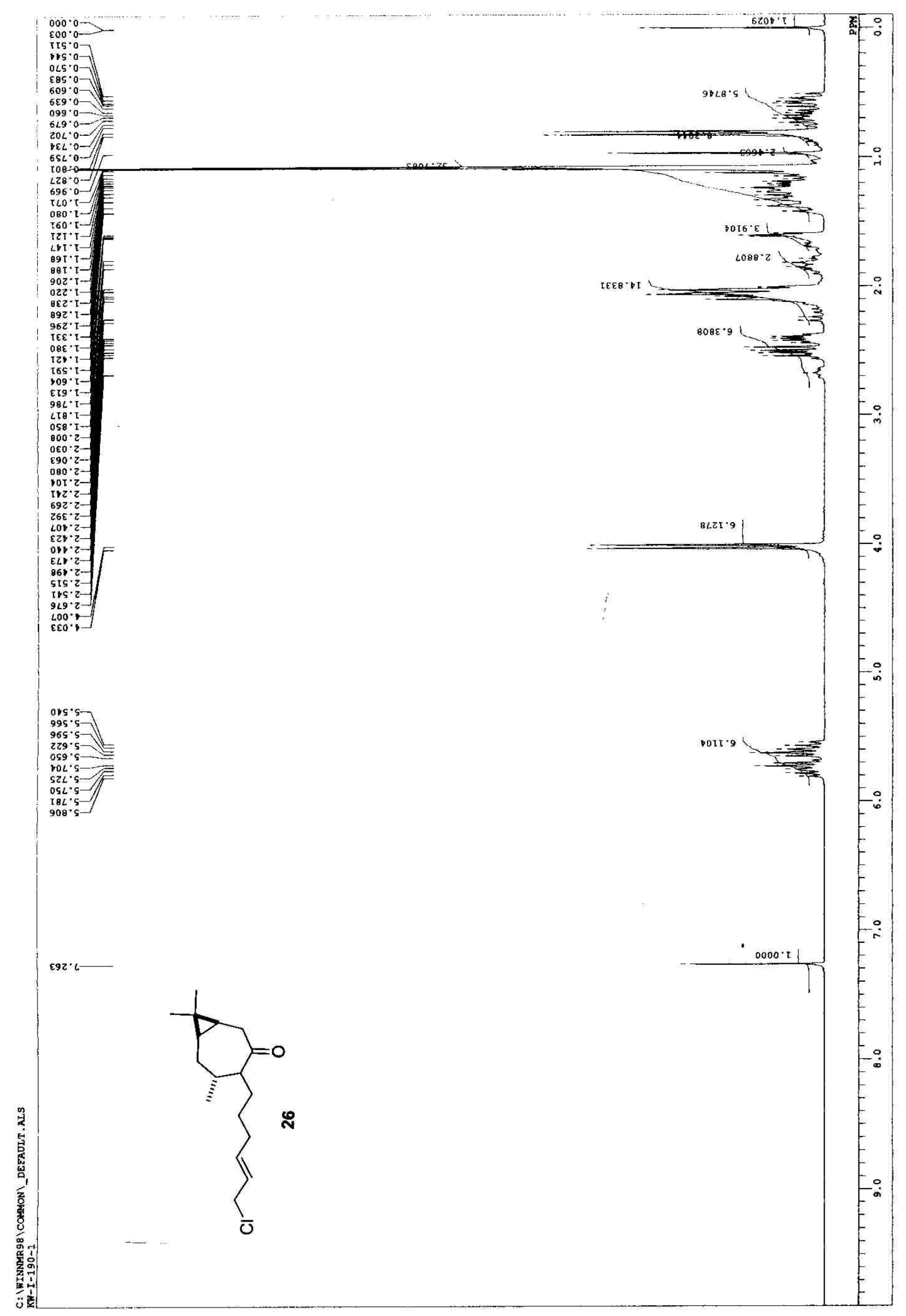



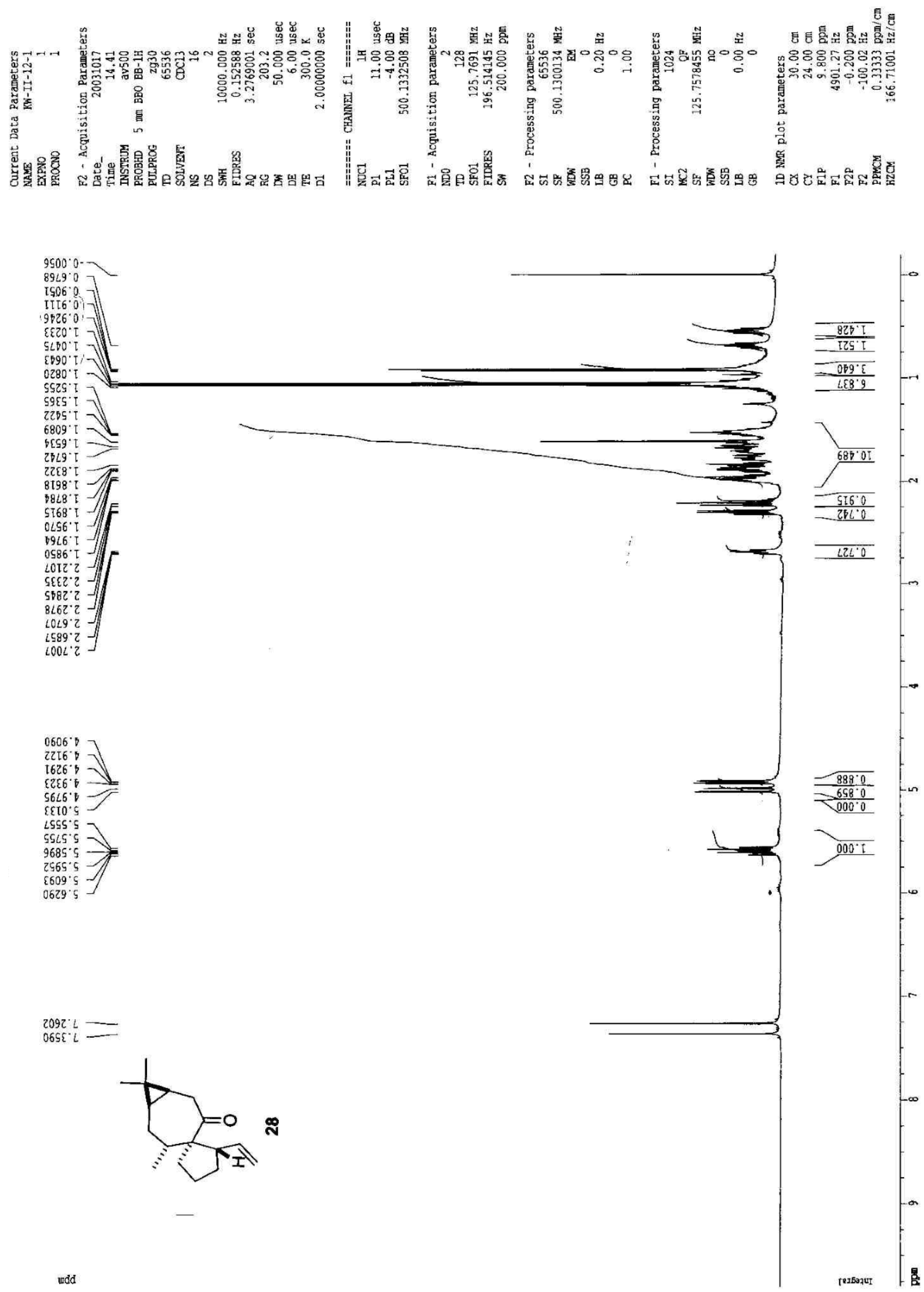


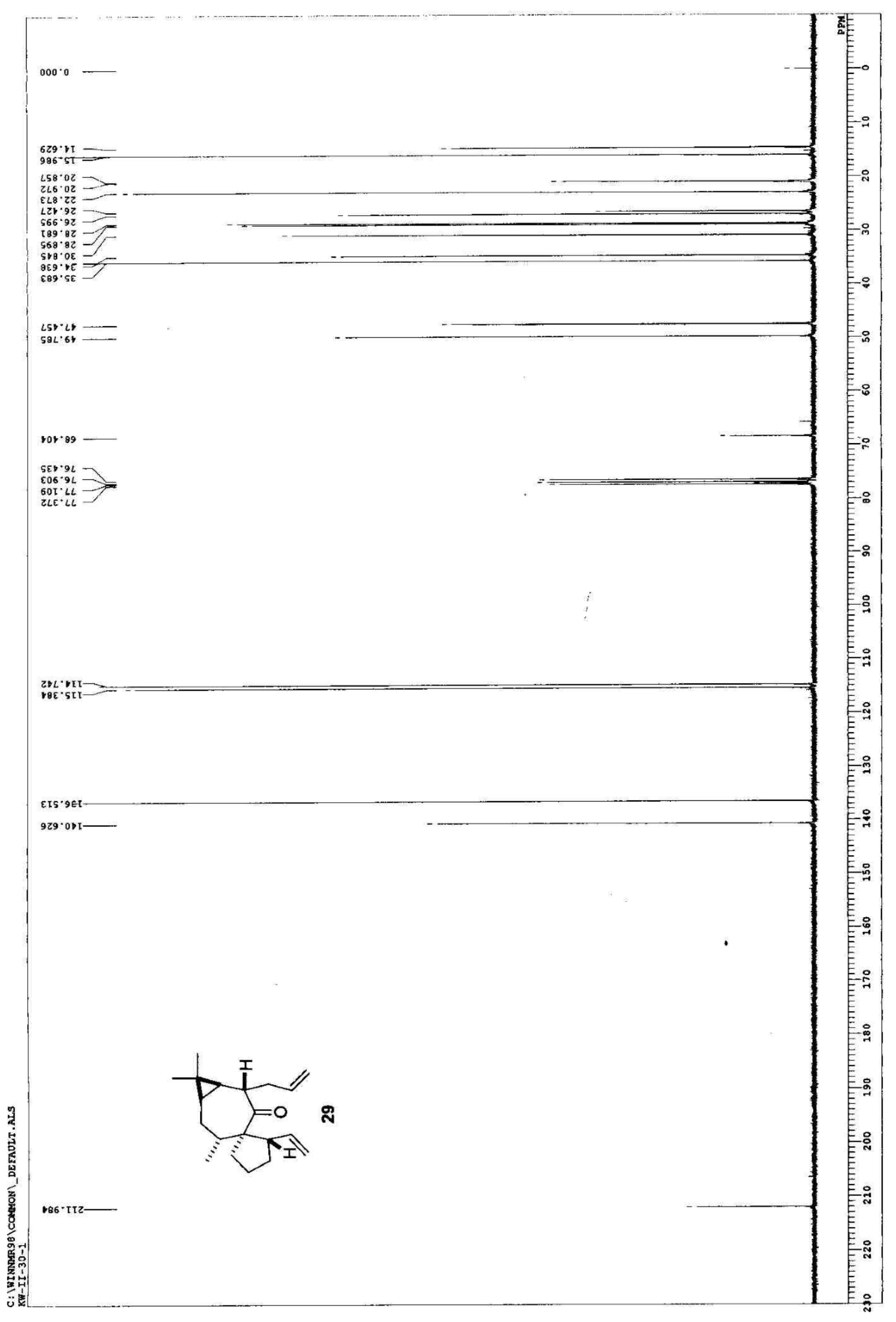



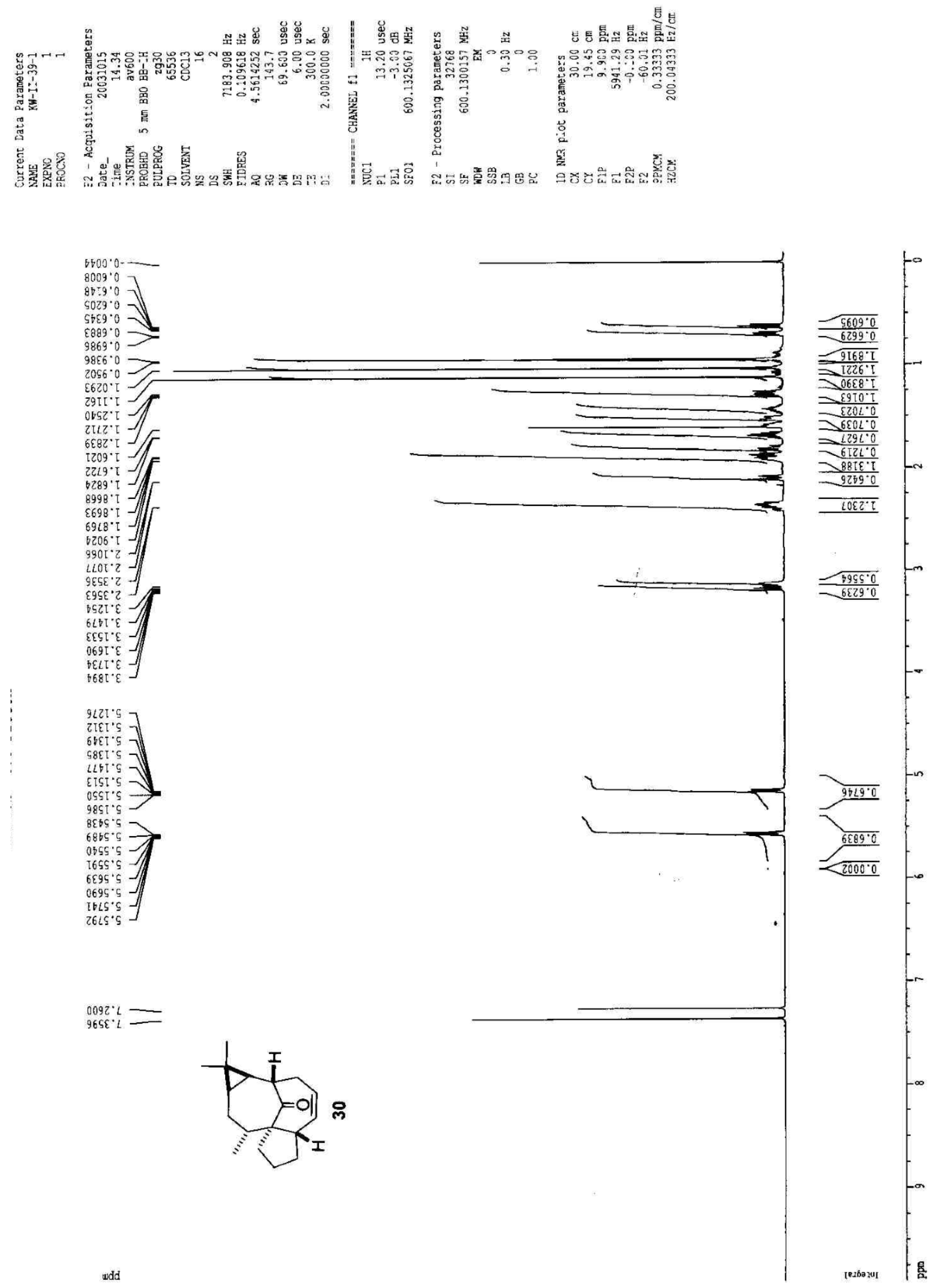


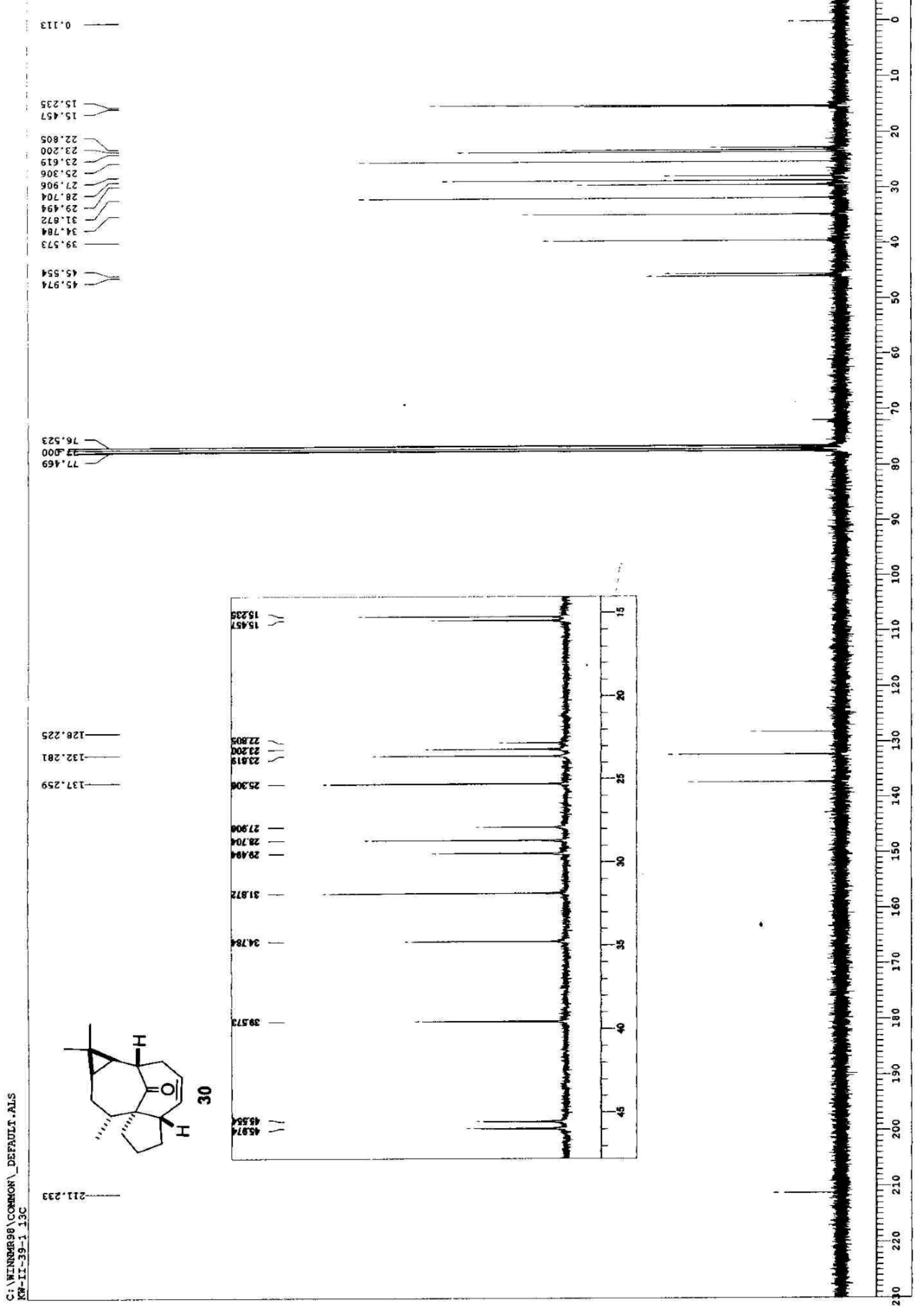




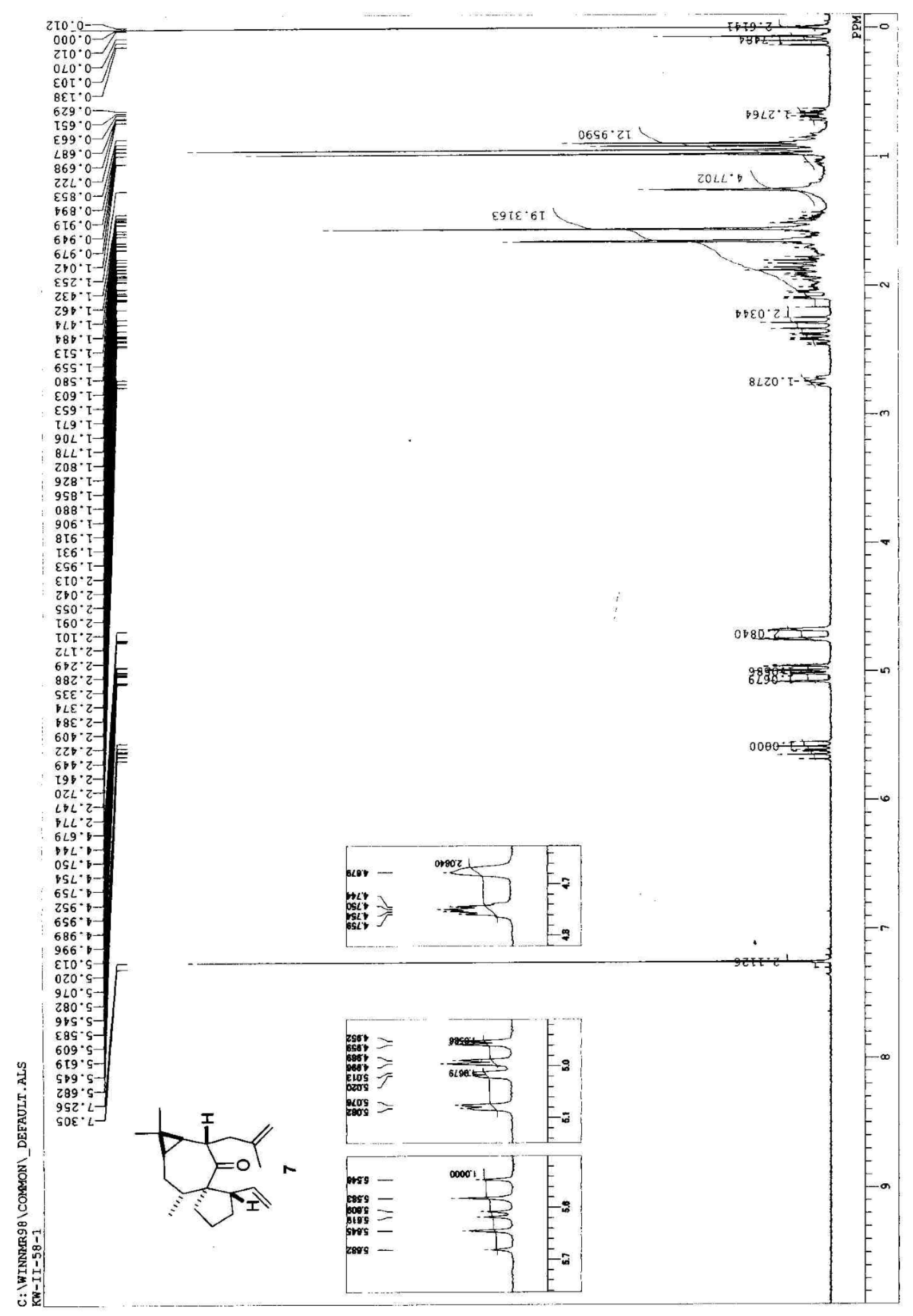




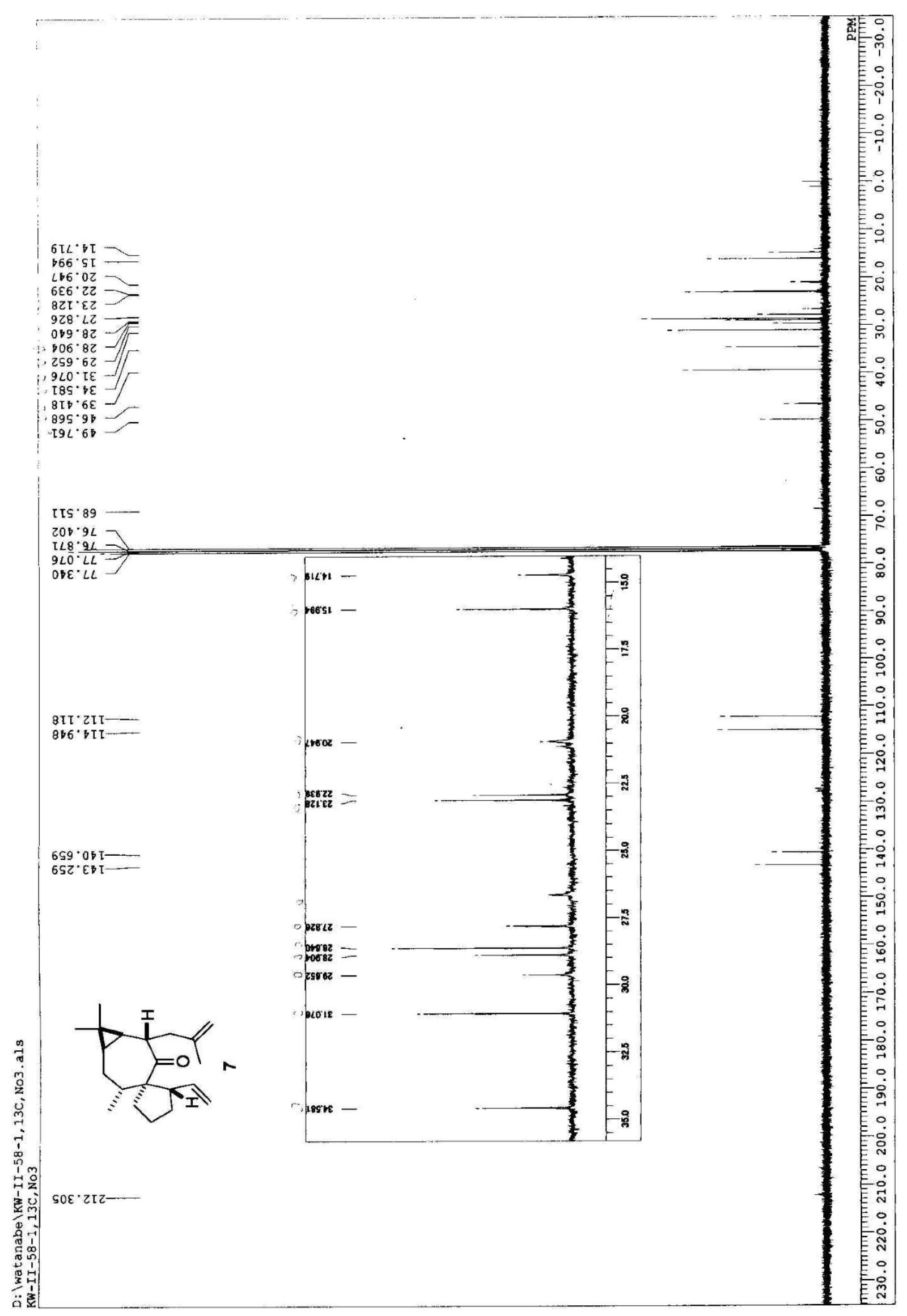



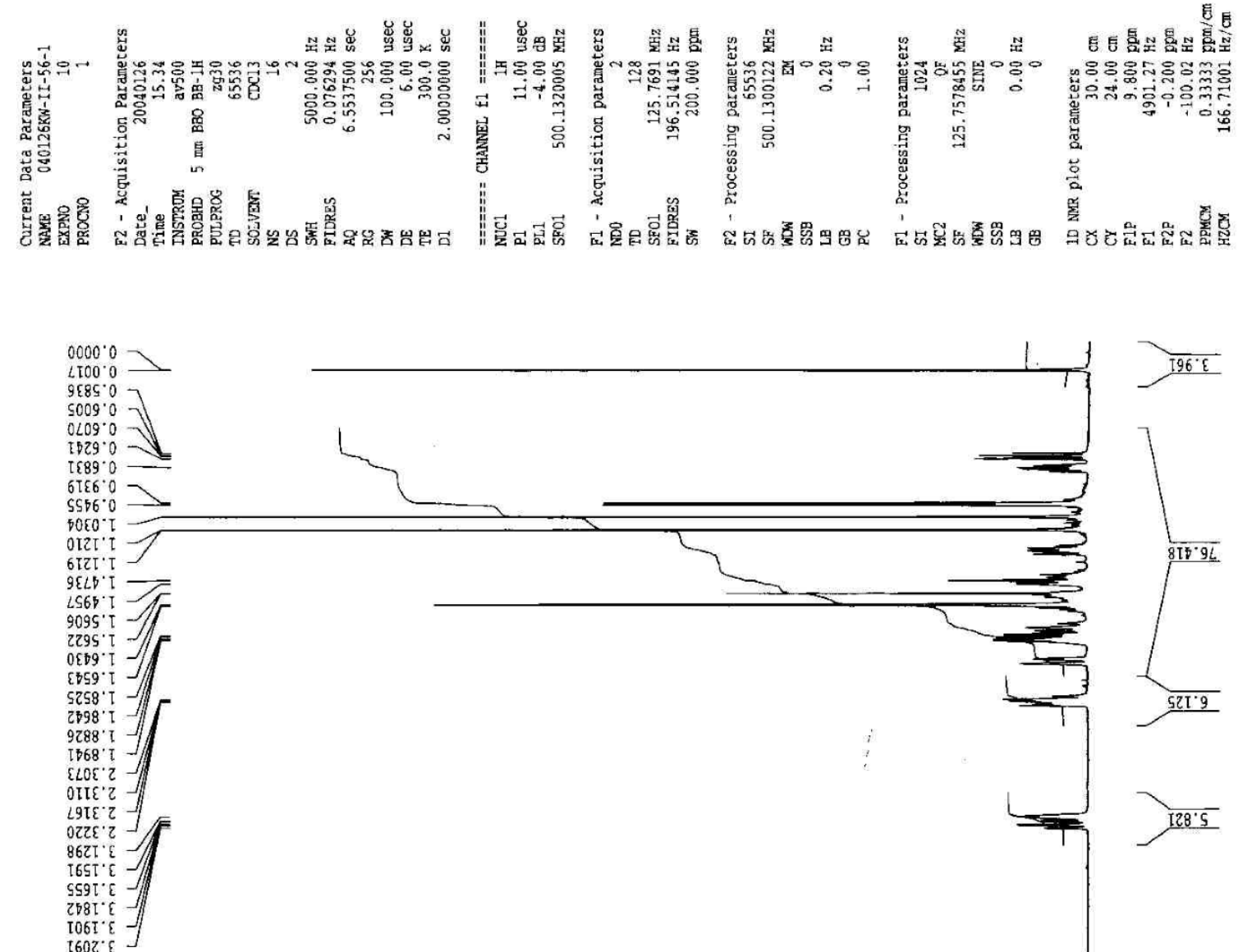

$0288^{\circ} \mathrm{C}$
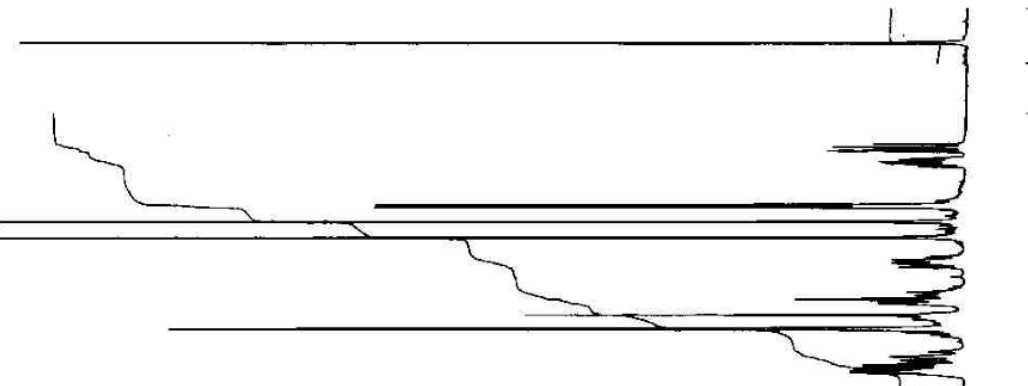

$\sqrt{196^{\circ} \varepsilon}$

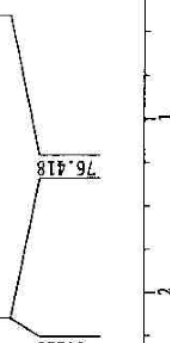

${ }_{90292 \cdot L}^{029}>$

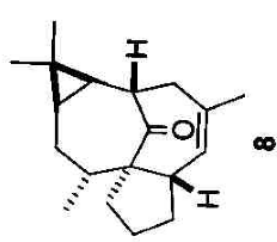




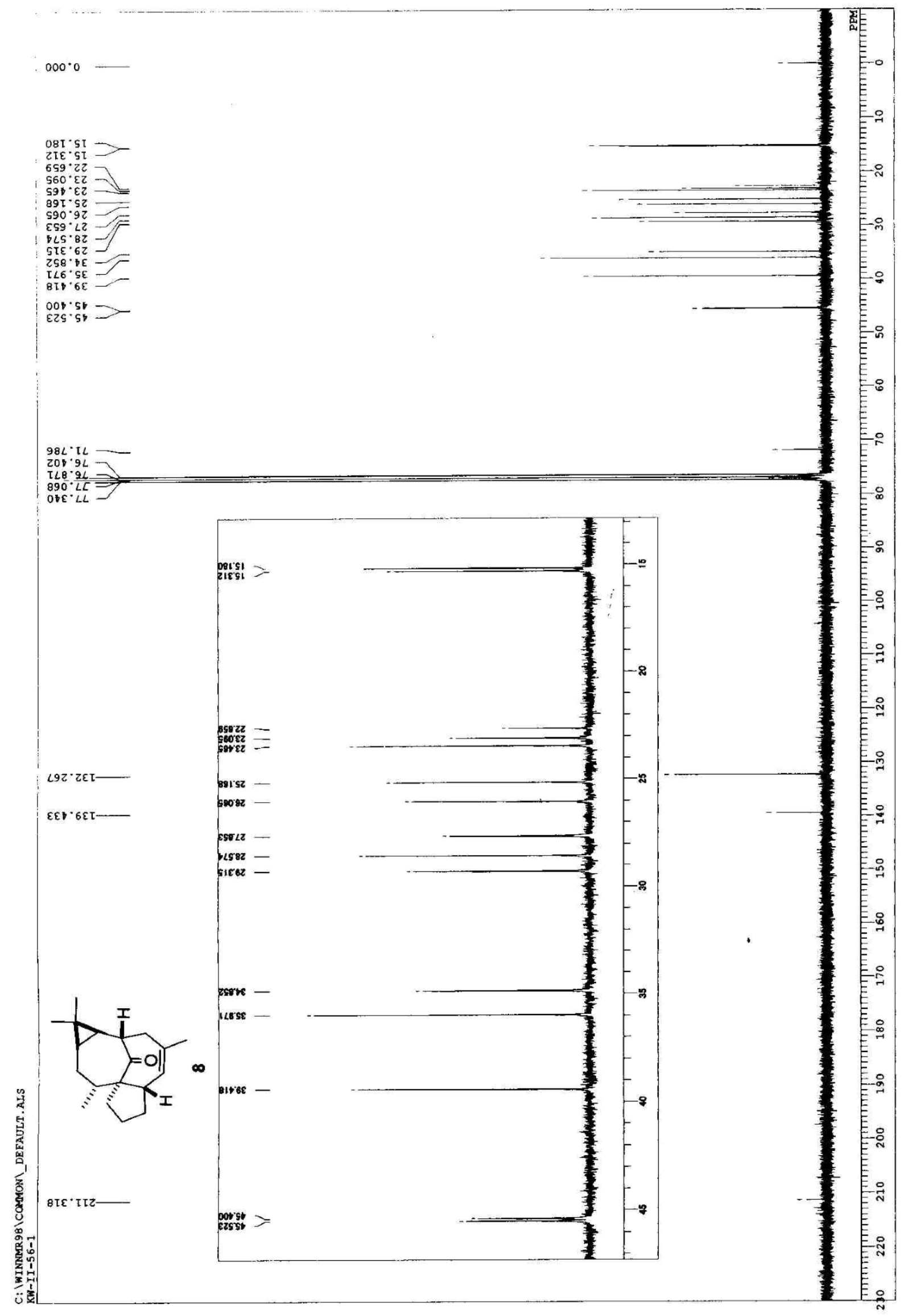



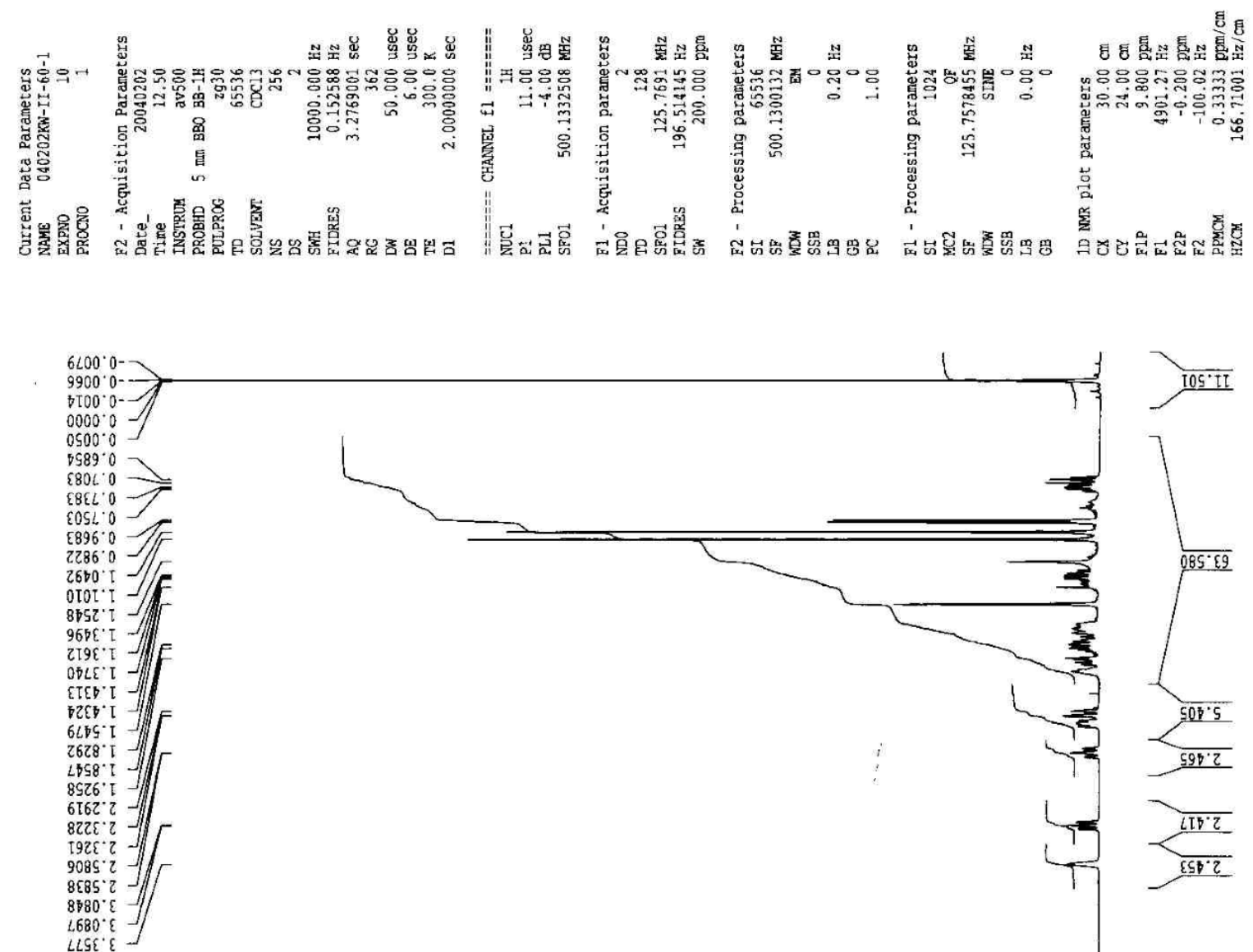

${ }_{9222 \cdot 9}^{6822 \cdot 9}>$

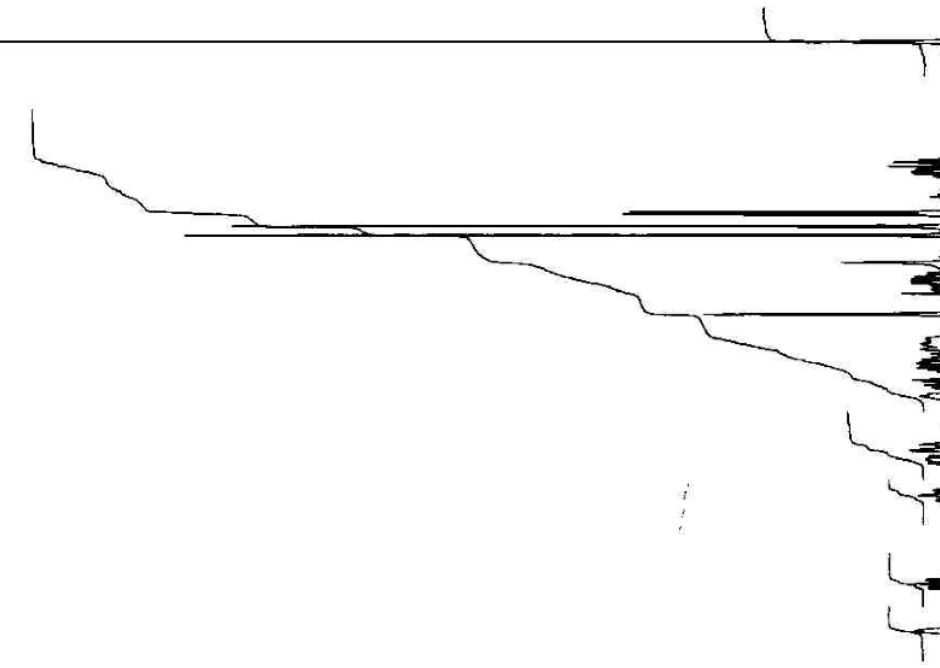

$00 . \pi I$
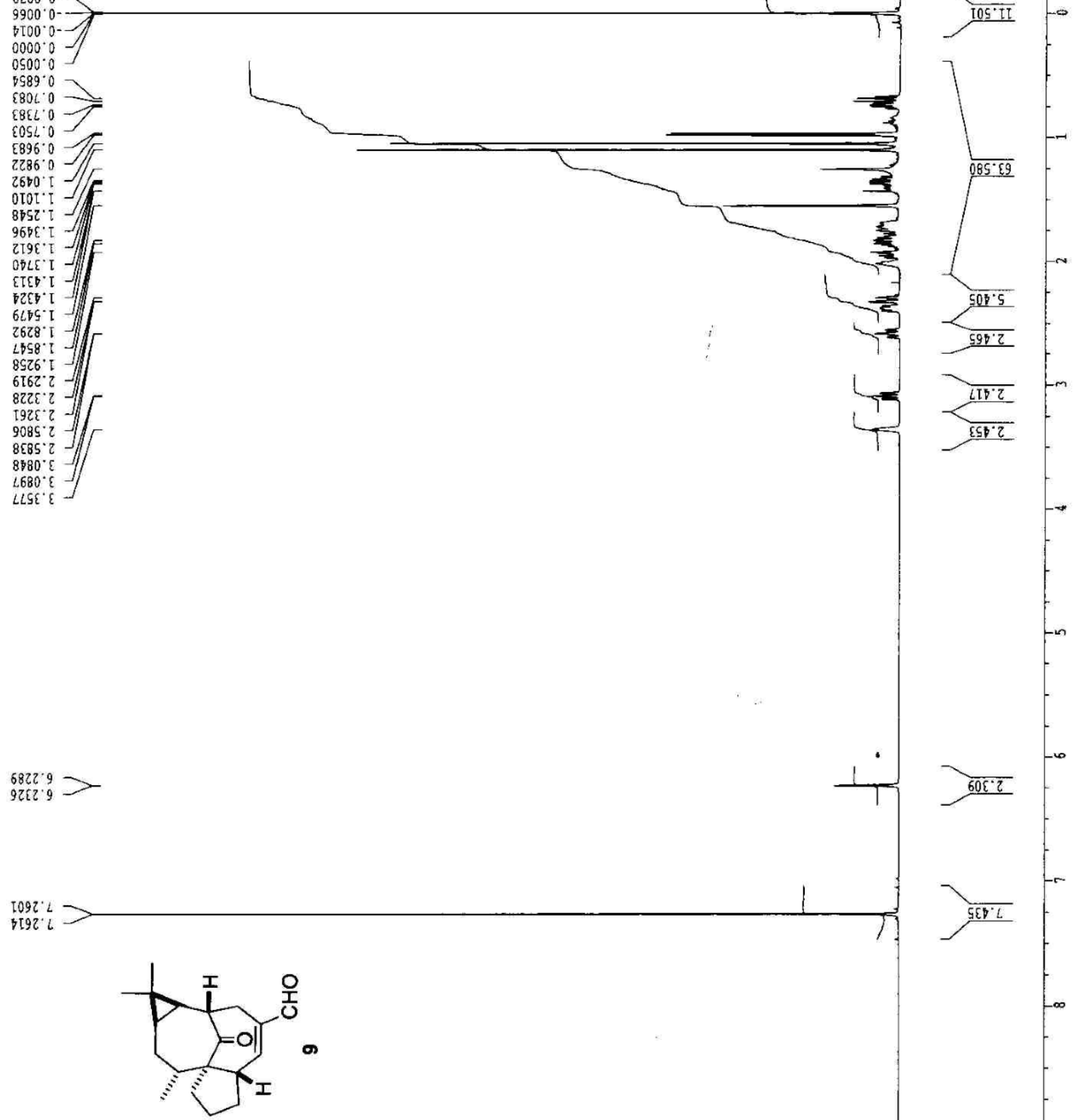

I8L2' 6

mda 
$656 \nabla 5$

G6E' 91

$\angle B L 2 己$

हSट हट

EEL ' $E$

$I \angle L S Z$

8टी 82

96E' 82

800.62

I6G 62

$\triangle E O O S$

$865^{\circ} \nabla \varepsilon$

$99 \angle 68$

$5 \angle 6 \circ D$

$5 \angle 8 \cdot 9 \mathrm{~V}$

$\nabla 56:$ :L

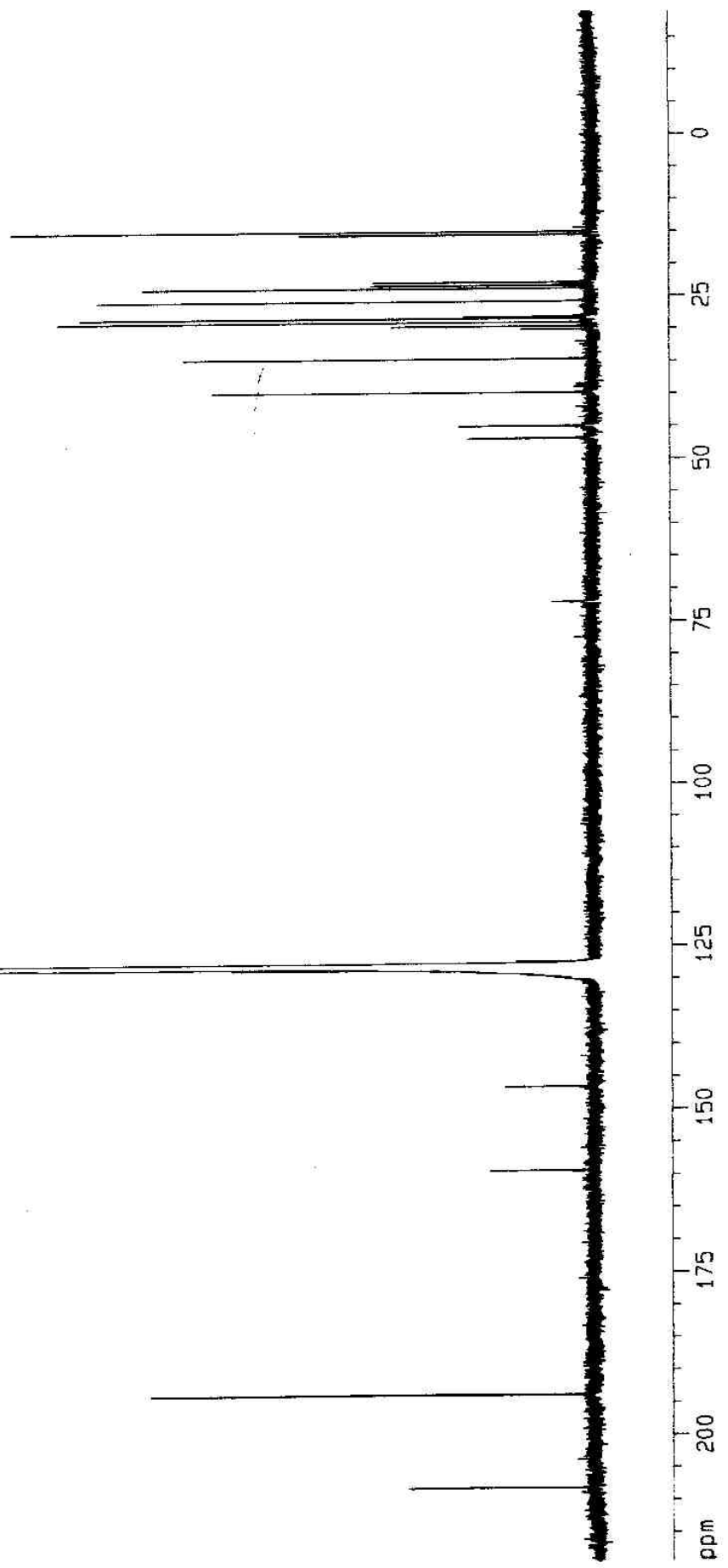

言

IDE $\angle 2 T$

8GL LCI

I8Q $L 2$ !

$866 \angle 2:-$

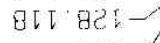

беट вटโ-

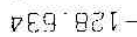

LOS $9 \nabla$ I

6EE 691

69l't:-..-

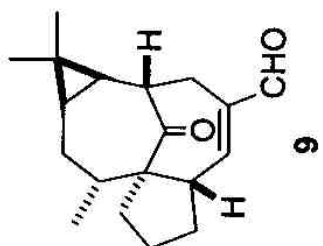

दर $902-$

พอบ 